\title{
Synthesis and Properties of a Novel Cross-Linked Electroactive Polymer Formed from a Bipolar Starburst Monomer
}

\author{
Jose Natera, Luis Otero, Fabiana D'Eramo, Leonides Sereno, and Fernando Fungo* \\ Departamento de Química, Universidad Nacional de Río Cuarto, Agencia Postal 3 (5800), \\ Río Cuarto, Argentina
}

Nung-Sen Wang, Yeun-Min Tsai,* and Ken-Tsung Wong*

Department of Chemistry, National Taiwan University, Taipei 106, Taiwan

Received August 16, 2008; Revised Manuscript Received November 10, 2008

\begin{abstract}
This Article describes the synthesis and physical properties of two bipolar starburst monomers: 1,3,5-tris $\{5$-(7-(carbazol-9-yl)-(9,9'-spirobifluoren-2-yl)-1,3,4-oxadiazol-2-yl $\}$ benzene (OXD-CBZ) and 1,3,5tris[7-diphenyl-(9,9'-spirobifluorene)-1,3,4-oxadiazoyl]benzene (OXD-DPA) featuring an electron-deficient tris(1,3,4oxadiazole)phenylene ring as an interior core bridged by rigid spirobifluorene units to terminal electroactive carbazole (CBZ) and diphenylamino (DPA) groups. The electronic absorption spectra of OXD-CBZ and OXDDPA depend only slightly on the solvent polarity, revealing that weak electronic coupling existed between the donor (CBZ and diphenylamino groups) and acceptor (1,3,4-oxadiazole) moieties in the ground state. In contrast, the emission spectra of these starburst bipolar molecules were strongly dependent on the solvent polarity, a phenomenon that we attribute to the polarized excited states generated through intramolecular charge transfer. The starburst monomer OXD-CBZ exhibited a redox gradient, where the peripheral CBZ moieties exhibited lower oxidation potentials than those of the fluorene units in the interior, allowing us to obtain a new electropolymerized macromolecule. The polymer films derived from OXD-CBZ exhibited good conductivity, reversible electrochemical processes, and stable color changes (from transparent to green to light blue) with high coloration efficiency upon electro-oxidation. The radical cations of OXD-CBZ were localized within the CBZ rings; the lack of substituents at the $\mathrm{C} 3$ and $\mathrm{C} 6$ positions of the CBZ unit led to the effective electrochemical cross-linking process. In contrast, electro-oxidation of OXD-DPA, which was end-capped with DPA groups, produced stable radical cations that did not undergo associated electrochemical reactions. The higher stability of the OXD-DPA radical cations through resonance stabilization with the fluorene ring impeded the progress of typical triphenylamine dimerization reactions.
\end{abstract}

\section{Introduction}

Dendrimers are regularly and hierarchically branched macromolecules presenting multiple chain ends emanating from a single core. Functional groups such as chromophores or electron donor/acceptor moieties can be positioned at designated positions, such as the core, focal points, the periphery, or even at each branching point of the dendritic structure. ${ }^{1,2}$ Dendrimer chemistry has rapidly expanded because of its significance in basic research and its practical applications. Particular attention has been focused on the preparation of photoactive and electroactive dendrimers, which are potentially useful as lightharvesting antennae, fluorescent chemosensors, and active materials for organic light-emitting diodes (OLEDs). ${ }^{3-10}$ A large number of synthetic dendrimers have featured an electroactive core or end-capping groups, ${ }^{3,11-17}$ resulting in novel hole and electron-transport dendritic materials $s^{4,5,18-22}$ that are key materials for improving the efficiency of advanced organic electronic or optoelectronic devices. In addition, dendrimers provide new opportunities for precisely placing constituents capable of transporting charge carriers by growing the various generations in a 3D manner. ${ }^{23,24}$ In our program of developing efficient charge-transport materials, we envisioned that hyperbranched polymeric materials formed electrochemically from tailor-made functional dendritic monomers might possess intriguing physical properties that would make them suitable for use in optoelectronics applications. Such a divergent strategy was previously applied for the synthesis of dendritic macromolecules using

* Corresponding authors. E-mail: ffungo@exa.unrc.edu.ar; kenwong@ ntu.edu.tw. electropolymerization ${ }^{25,26}$ and living polymerization ${ }^{27}$ technologies, which resulted in highly branched dendrimers emanating from a functionalized core that possessed interesting structural features and novel properties. The polymerizable peripherals of dendritic monomers can react not only intermolecularly to give the highly branched macromolecules with globular architecture but also intramolecularly (if positioned appropriately) to give nanoparticles featuring conjugated polymer shells. ${ }^{28}$

Molecules containing electroactive carbazole (CBZ) and diphenylamine (DPA) moieties are interesting materials because their redox and photophysical properties make them suitable for use as hole carriers in optoelectronic devices. ${ }^{29-33}$ The electro-optical properties of dendrimeric ${ }^{8-17,34,35}$ and polymeric thin films deposited on flat conducting substrates through the electropolymerization of polyfluorene precursors containing pendent electroactive CBZ and DPA units have been reported. $^{20-22,36}$ The large differences in oxidation potential between fluorene rings and CBZ and DPA units ensures that the electropolymerization selectively occurs on the pendant groups without affecting the fluorene backbone. ${ }^{20-22,37,38}$ Furthermore, oligofluorenes have recently attracted much attention because of their chemical and thermal stabilities and the possibility of tailoring the physical properties via the introduction of functional substituents at the C9 position. $^{20,21,39-41}$ Remarkably, polyfluorenes and oligofluorenes end-capped with hole-transporting moieties improve the performance of OLEDs. ${ }^{3,22,42-46}$ Therefore, we suspected that combining these structural features into a starburst monomer might be a potentially useful approach toward creating new macromolecules that display improved electro-optical properties. 
In this Article, we describe the synthesis and properties of two bipolar starburst monomers: 1,3,5-tris $\{5$-(7-(carbazol-9-yl)-(9,9'spirobifluoren-2-yl)-1,3,4-oxadiazol-2-yl \}benzene (OXD-CBZ) and 1,3,5-tris[7-diphenyl-(9,9'-spirobifluorene)-1,3,4-oxadiazoyl]benzene (OXD-DPA) featuring an electron-deficient tris1,3,4-oxadiazole phenylene ring as an interior core bridged by a rigid spirobifluorene unit end-capped with electroactive DPA and CBZ groups (Scheme 1). The new structures exhibit redox gradients in which the peripheral CBZ moieties exhibit lower oxidation potentials than do the fluorene and 1,3,4-oxadiazole substituted benzene units in the interior. This molecular design strategy allowed us to obtain a new electropolymerized macromolecule that displays interesting electronic and optical properties: good conductivity, reversible electrochemical processes, and stable color changes with high coloration efficiency. This Article provides one of only a few examples of using starburst monomers to form electrochemically active polymers.

\section{Experimental Procedures}

2.1. Materials. 2-Bromo-7-(carbazol-9-yl)-9,9'-spirobifluorene (1) was prepared by the reported procedure. ${ }^{20}$ Commercially available reagents were used as received without further purification: Cuprous cyanide $(\mathrm{CuCN})$ (Acros, 99\%), N,N-dimethylformamide (DMF) (Acros, 99.5\%), ammonium hydroxide $\left(\mathrm{NH}_{4} \mathrm{OH}\right)$ (Acros, 28 to 30 wt \%), methylene chloride $\left(\mathrm{CH}_{2} \mathrm{Cl}_{2}\right)$ (Tedia), magnesium sulfate $\left(\mathrm{MgSO}_{4}\right)$ (Acros, $97 \%$,), silica gel $\left(\mathrm{SiO}_{2}\right)$ (Merk, 230-400 mesh), benzene (Tedia), acetonitrile $\left(\mathrm{CH}_{3} \mathrm{CN}\right)$ (Acros, $\left.99.5 \%\right)$, sodium azide (Acros, 99\%), ammonium chloride (Acros, 99\%), 1,3,5-benzenetricarboxylic acid chloride (Aldrich, 99\%), toluene (Acros, 99\%), tetrahydrofuran (THF) (Tedia), tri-tert-butylphosphine (Acros, 95\%), palladium acetate $\left(\mathrm{Pd}(\mathrm{OAc})_{2}\right)$ (Acros, $47.5 \%$ $\mathrm{Pd})$, diphenylamine (Acros, $99 \%)$, sodium tert-butoxide $\left(\mathrm{NaO}^{t} \mathrm{Bu}\right)$ (Acros, 98\%), ferrocene (Acros, 98\%), and tetra $n$-butyl ammonium perchlorate (TBAP) (Fluka, 99\%). All reactions were performed under argon and were magnetically stirred. Solvents were distilled from the appropriate drying agent prior to use: THF from sodium and benzophenone (Acros, 98\%), toluene from sodium, DMF from potassium hydride, and $\mathrm{CH}_{2} \mathrm{Cl}_{2}$ from $\mathrm{P}_{2} \mathrm{O}_{5}$. All reactions were monitored by TLC with Macherey-Nagel precoated aluminum foil sheets $\left(0.20 \mathrm{~mm}\right.$ with fluorescent indicator $\left.\mathrm{UV}_{254}\right)$. Compounds were visualized with UV light at either 254 or $365 \mathrm{~nm}$.

2.2. Instrumentation. Melting points were measured by MelTemp II (Laboratory Devices) without calibration. Infrared (IR) spectra were recorded as neat films or $\mathrm{KBr}$ pellets on a Nicolet FT-IR spectrometer. ${ }^{1} \mathrm{H}$ NMR and ${ }^{13} \mathrm{C}$ NMR in $\mathrm{CDCl}_{3}$ or DMSO$d_{6}$ were recorded using a Bruker (Avance-400) or Varian (Unity Plus 400) spectrometer at 400 and $100 \mathrm{MHz}$, respectively. Lowand high-resolution mass spectra were recorded using a Jeol SX$102 \mathrm{~A}$ spectrometer in fast-atom bombardment (FAB) mode, a Shimadzu IT-TOF mass spectrometer in electronspray ionization (ESI) mode, or a Finnigan MAT 95S apparatus in electron ionization (EI) mode. Microanalyses were carried out on a Heraeus VarioIII$\mathrm{NCH}$ analyzer. Ultraviolet (UV) spectra were measured with a Hitachi U-2800A spectrophotometer. Photoluminescence (PL) was measured with a Hitachi F-4500 fluorescence spectrophotometer. PL quantum yields were measured using a Hamamatsu C9920 integration sphere that gives anthracene in ethanol with a quantum yield of $27 \%$.

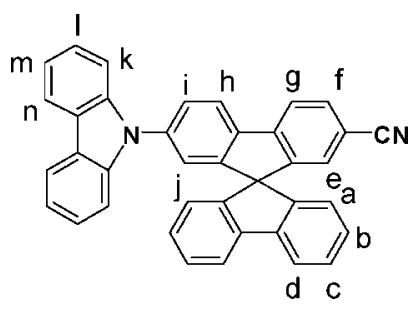

2.3. Synthesis. 2.3.1. 2-(Carbazol-9-yl)-7-cyano-9,9'-spirobifluorene (2). A mixture of the 2-bromo-7-(carbazol-9-yl)-9, $9^{\prime}$-spirobifluorene $(\mathbf{1})^{20}(1.2 \mathrm{~g}, 2.4 \mathrm{mmol})$ and $\mathrm{CuCN}(0.43 \mathrm{~g}, 4.8 \mathrm{mmol})$ in dry DMF (12 mL) was heated under reflux for $48 \mathrm{~h}$ under argon. After cooling to room temperature, the solution was quenched with ammonium hydroxide $(28 \%, 70 \mathrm{~mL})$ and $\mathrm{CH}_{2} \mathrm{Cl}_{2}(100 \mathrm{~mL})$. The organic layer was dried $\left(\mathrm{MgSO}_{4}\right)$, filtered, and concentrated in vacuo. The residue was purified through column chromatography $\left(\mathrm{SiO}_{2}\right.$; hexane/ethyl acetate, 85:15) to afford 2 as a yellow solid (0.91 g, 82\%). mp 336-338 ${ }^{\circ} \mathrm{C}$. IR (neat) $v\left(\mathrm{~cm}^{-1}\right): 3065,2217$ $(-\mathrm{CN}), 1613(\mathrm{C}=\mathrm{C}), 1448 .{ }^{1} \mathrm{H} \mathrm{NMR}\left(\mathrm{CDCl}_{3}, 400 \mathrm{MHz}, \delta\right): 6.81$ $\left(\mathrm{d}, J=7.5 \mathrm{~Hz}, 2 \mathrm{H}, \mathrm{H}_{\mathrm{a}}\right), 6.96\left(\mathrm{~s}, 1 \mathrm{H}, \mathrm{H}_{\mathrm{j}}\right), 7.05\left(\mathrm{~s}, 1 \mathrm{H}, \mathrm{H}_{\mathrm{e}}\right), 7.14-7.23$ $\left(\mathrm{m}, 6 \mathrm{H}, \mathrm{H}_{\mathrm{b}, \mathrm{m}, \mathrm{k}}\right), 7.28\left(\mathrm{t}, J=7.5 \mathrm{~Hz}, 2 \mathrm{H}, \mathrm{H}_{\mathrm{l}}\right), 7.39(\mathrm{t}, J=7.5 \mathrm{~Hz}$, $\left.2 \mathrm{H}, \mathrm{H}_{\mathrm{c}}\right), 7.64\left(\mathrm{~d}, J=7.5 \mathrm{~Hz}, 1 \mathrm{H}, \mathrm{H}_{\mathrm{i}}\right), 7.71(\mathrm{~d}, J=7.5 \mathrm{~Hz}, 1 \mathrm{H}$, $\left.\mathrm{H}_{\mathrm{f}}\right), 7.81\left(\mathrm{~d}, J=7.5 \mathrm{~Hz}, 2 \mathrm{H}, \mathrm{H}_{\mathrm{d}}\right), 7.97\left(\mathrm{~d}, J=7.5 \mathrm{~Hz}, 1 \mathrm{H}, \mathrm{H}_{\mathrm{h}}\right)$, $8.03\left(\mathrm{~d}, J=7.5 \mathrm{~Hz}, 2 \mathrm{H}, \mathrm{H}_{\mathrm{n}}\right), 8.09\left(\mathrm{~d}, J=7.5 \mathrm{~Hz}, 1 \mathrm{H}, \mathrm{H}_{\mathrm{g}}\right) .{ }^{13} \mathrm{C}$ NMR $\left(\mathrm{CDCl}_{3}, 100 \mathrm{MHz}, \delta\right): 65.8,109.4,111.0,118.8,120.0,120.2$, 120.4, 120.6, 122.1, 122.6, 123.3, 123.6, 125.8, 126.6, 127.8, 128.1, 128.4, 132.2, 138.4, 138.7, 140.2, 141.6, 145.1, 146.3, 149.9, 151.5. MS (FAB) $\mathrm{m} / z$ (relative intensity): $506\left(\mathrm{M}^{+}, 17\right), 406$ (14), 307 (100), 289 (47). HRMS (FAB ${ }^{+}$): calcd for $\mathrm{C}_{38} \mathrm{H}_{22} \mathrm{~N}_{2}$ 506.1783; found, 506.1780; Anal. Calcd for $\mathrm{C}_{38} \mathrm{H}_{22} \mathrm{~N}_{2}$ : C, 90.09; H, 4.38; N, 5.53. Found: C, 89.54; H, 4.59; N, 5.45.

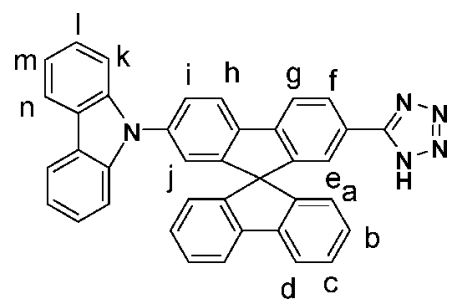

2.3.2. 2-(Carbazol-9-yl)-7-tetrazol-1-yl-9,9'-spirobifluorene (3). A mixture of the nitrile $2(0.55 \mathrm{~g}, 1.1 \mathrm{mmol})$, sodium azide $(0.14$ $\mathrm{g}, 2.2 \mathrm{mmol})$, and ammonium chloride $(0.12 \mathrm{~g}, 2.2 \mathrm{mmol})$ in dry DMF (12 mL) was heated at $120{ }^{\circ} \mathrm{C}$ (oil bath) for $48 \mathrm{~h}$ under argon. After cooling to room temperature, the solution was added dropwise to $3 \mathrm{~N}$ hydrochloric acid $(60 \mathrm{~mL})$ and then vigorously stirred for $10 \mathrm{~min}$. The solid was collected by filtration; the filter cake was washed with water and dried in vacuo to afford 3 as a yellow solid $(0.55 \mathrm{~g}, 92 \%)$. mp 263-264 ${ }^{\circ} \mathrm{C}$. IR $(\mathrm{KBr}) v\left(\mathrm{~cm}^{-1}\right): 3457(\mathrm{~N}-\mathrm{H}), 3047,1639(\mathrm{C}=\mathrm{C}), 1634$ $(\mathrm{C}=\mathrm{C}), 1434 .{ }^{1} \mathrm{H}$ NMR (DMSO- $\left.d_{6}, 400 \mathrm{MHz}, \delta\right): 6.75(\mathrm{~s}, 1 \mathrm{H}$, $\left.\mathrm{H}_{\mathrm{j}}\right), 6.84,\left(\mathrm{~d}, J=7.5 \mathrm{~Hz}, 2 \mathrm{H}, \mathrm{H}_{\mathrm{a}}\right), 7.10\left(\mathrm{~d}, J=7.5 \mathrm{~Hz}, 2 \mathrm{H}, \mathrm{H}_{\mathrm{k}}\right)$, $7.18-7.24\left(\mathrm{~m}, 4 \mathrm{H}, \mathrm{H}_{\mathrm{b}, \mathrm{m}}\right), 7.29\left(\mathrm{t}, J=7.5 \mathrm{~Hz}, 2 \mathrm{H}, \mathrm{H}_{\mathrm{l}}\right), 7.36(\mathrm{~s}$, $\left.1 \mathrm{H}, \mathrm{H}_{\mathrm{e}}\right), 7.44\left(\mathrm{t}, J=7.5 \mathrm{~Hz}, 2 \mathrm{H}, \mathrm{H}_{\mathrm{c}}\right), 7.74(\mathrm{~d}, J=7.5 \mathrm{~Hz}, 1 \mathrm{H}$, $\left.\mathrm{H}_{\mathrm{i}}\right), 8.03\left(\mathrm{~d}, J=8 \mathrm{~Hz}, 2 \mathrm{H}, \mathrm{H}_{\mathrm{d}}\right), 8.15\left(\mathrm{~d}, J=8 \mathrm{~Hz}, 2 \mathrm{H}, \mathrm{H}_{\mathrm{n}}\right)$, $8.20\left(\mathrm{~d}, J=8 \mathrm{~Hz}, 1 \mathrm{H}, \mathrm{H}_{\mathrm{f}}\right), 8.39\left(\mathrm{~d}, J=8 \mathrm{~Hz}, 1 \mathrm{H}, \mathrm{H}_{\mathrm{h}}\right), 8.43(\mathrm{~d}$, $J=8 \mathrm{~Hz}, 1 \mathrm{H}, \mathrm{H}_{\mathrm{g}}$ ). ${ }^{13} \mathrm{C}$ NMR (DMSO- $\left.d_{6}, 100 \mathrm{MHz}, \delta\right): 65.4$, $109.0,120.0,120.4,120.7,121.1,121.6,121.8,122.5,122.9$, $123.3,126.0,126.5,127.1,128.1,128.2,136.9,139.2,139.5$, 141.1, 143.0, 146.7, 149.3, 150.1. FAB MS $\mathrm{m} / \mathrm{z}$ (relative intensity): $550\left(\mathrm{M}^{+}, 65\right), 521$ (22), 506 (21), 391 (94), 307 (100), 289 (66). HRMS $\left(\mathrm{FAB}^{+}\right):[\mathrm{M}+\mathrm{H}]^{+}$calcd for $\mathrm{C}_{38} \mathrm{H}_{24} \mathrm{~N}_{5}$, 550.2032; found, 550.2022.

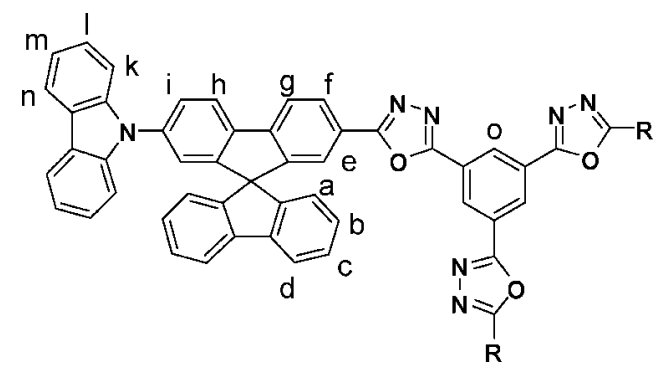

2.3.3. 1,3,5-Tris (5-(7-(carbazol-9-yl)-(9,9'-spirobifluoren-2-yl)-1,3,4oxadiazol-2-yllbenzene (OXD-CBZ). 1,3,5-Benzenetricarboxylic acid chloride $(0.10 \mathrm{~g}, 0.38 \mathrm{mmol})$ was added in one portion to a solution 
of the tetrazole $3(0.68 \mathrm{~g}, 1.2 \mathrm{mmol})$ in dry toluene $(20 \mathrm{~mL})$. The resulting mixture was heated under reflux for $17 \mathrm{~h}$ under argon. After cooling to room temperature, the solution was concentrated in vacuo. The crude product was recrystallized from hexane/THF to afford OXD-CBZ as a yellow solid (0.86 g, 86\%). mp 308-310 ${ }^{\circ} \mathrm{C}$. IR (neat) $v\left(\mathrm{~cm}^{-1}\right): 3065,2986,1607(\mathrm{C}=\mathrm{C}), 1540,1448 .{ }^{1} \mathrm{H}$ NMR $\left(\mathrm{CDCl}_{3}, 400 \mathrm{MHz}, \delta\right): 6.86\left(\mathrm{~d}, J=8 \mathrm{~Hz}, 6 \mathrm{H}, \mathrm{H}_{\mathrm{a}}\right), 6.94(\mathrm{~s}$, $\left.3 \mathrm{H}, \mathrm{H}_{\mathrm{j}}\right), 7.14-7.19\left(\mathrm{~m}, 18 \mathrm{H}, \mathrm{H}_{\mathrm{b}, \mathrm{k}, \mathrm{m}}\right), 7.24\left(\mathrm{t}, J=7 \mathrm{~Hz}, 6 \mathrm{H}, \mathrm{H}_{\mathrm{l}}\right)$, $7.40\left(\mathrm{t}, J=8 \mathrm{~Hz}, 6 \mathrm{H}, \mathrm{H}_{\mathrm{c}}\right), 7.66\left(\mathrm{~s}, 3 \mathrm{H}, \mathrm{H}_{\mathrm{e}}\right), 7.67(\mathrm{~d}, J=10 \mathrm{~Hz}$, $\left.3 \mathrm{H}, \mathrm{H}_{\mathrm{i}}\right), 7.86\left(\mathrm{~d}, J=7 \mathrm{~Hz}, 6 \mathrm{H}, \mathrm{H}_{\mathrm{d}}\right), 8.00\left(\mathrm{~d}, J=8 \mathrm{~Hz}, 6 \mathrm{H}, \mathrm{H}_{\mathrm{n}}\right)$, $8.12\left(\mathrm{~d}, J=8 \mathrm{~Hz}, 3 \mathrm{H}, \mathrm{H}_{\mathrm{f}}\right), 8.16\left(\mathrm{~d}, J=8 \mathrm{~Hz}, 3 \mathrm{H}, \mathrm{H}_{\mathrm{h}}\right), 8.28(\mathrm{~d}, J$ $\left.=7 \mathrm{~Hz}, 3 \mathrm{H}, \mathrm{H}_{\mathrm{g}}\right), 8.70\left(\mathrm{~s}, 3 \mathrm{H}, \mathrm{H}_{\mathrm{o}}\right) \cdot{ }^{13} \mathrm{C} \mathrm{NMR}\left(\mathrm{CDCl}_{3}, 100 \mathrm{MHz}\right.$, $\delta): 66.0,109.5,119.9,120.1,120.5,120.7,121.9,122.4,122.5$, 123.0, 123.3, 123.7, 125.8, 126.5, 127.1, 127.4, 128.0, 128.3, 138.2, $138.9,140.2,141.8,144.9,146.8,150.0,151.8,162.4,165.3 . \mathrm{MS}$ (FAB) $\mathrm{m} / \mathrm{z}$ (relative intensity): $1721\left([\mathrm{M}+\mathrm{H}]^{+}, 1\right), 1592(1), 1089$ (7), 307 (100), 289 (50). Anal. Calcd for $\mathrm{C}_{123} \mathrm{H}_{69} \mathrm{~N}_{9} \mathrm{O}_{3} \mathrm{C}$, 85.84; H, 4.04; N, 7.33. Found C, 85.64; H, 4.01; N, 7.13.

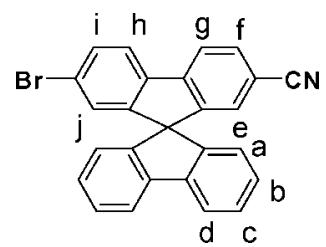

2.3.4. 2-Bromo-7-cyano-9,9'-spirobifluorene (4). A mixture of 2,7dibromo-9,9'-spirobifluorene (4.00 g, $8.37 \mathrm{mmol})$ and $\mathrm{CuCN}(0.50$ $\mathrm{g}, 5.58 \mathrm{mmol})$ in dry DMF $(56 \mathrm{~mL})$ was heated under reflux for $15 \mathrm{~h}$ under argon. After cooling to room temperature, the solution was partitioned between $\mathrm{NH}_{4} \mathrm{OH}_{(\mathrm{aq})}(28 \%)$ and $\mathrm{CH}_{2} \mathrm{Cl}_{2}$. The organic layer was dried $\left(\mathrm{MgSO}_{4}\right)$, filtered, and concentrated in vacuo. The residue was purified through column chromatography $\left(\mathrm{SiO}_{2}\right.$ : hexane/ ethyl acetate, 95:5) to afford $\mathbf{4}$ as a yellow solid (1.85 g, 79\%). mp $238-240{ }^{\circ} \mathrm{C}$. IR (neat) $v\left(\mathrm{~cm}^{-1}\right): 3066,3036,2221(-\mathrm{CN}), 1603$ $(\mathrm{C}=\mathrm{C}), 1457 .{ }^{1} \mathrm{H}$ NMR $\left(\mathrm{CDCl}_{3}, 400 \mathrm{MHz}, \delta\right): 6.67(\mathrm{~d}, J=7.5$ $\left.\mathrm{Hz}, 2 \mathrm{H}, \mathrm{H}_{\mathrm{a}}\right), 6.89\left(\mathrm{~d}, J=1.5 \mathrm{~Hz}, 1 \mathrm{H}, \mathrm{H}_{\mathrm{j}}\right), 6.96\left(\mathrm{~s}, 1 \mathrm{H}, \mathrm{H}_{\mathrm{e}}\right), 7.14$ $\left(\mathrm{t}, J=7.5 \mathrm{~Hz}, 2 \mathrm{H}, \mathrm{H}_{\mathrm{b}}\right), 7.41\left(\mathrm{t}, J=7.5 \mathrm{~Hz}, 2 \mathrm{H}, \mathrm{H}_{\mathrm{c}}\right), 7.53(\mathrm{dd}, J$ $\left.=7.5,1.5 \mathrm{~Hz}, 1 \mathrm{H}, \mathrm{H}_{\mathrm{i}}\right), 7.64\left(\mathrm{~d}, J=7.5 \mathrm{~Hz}, 1 \mathrm{H}, \mathrm{H}_{\mathrm{f}}\right), 7.73(\mathrm{~d}, J=$ $\left.7.5 \mathrm{~Hz}, 1 \mathrm{H}, \mathrm{H}_{\mathrm{h}}\right), 7.84-7.88\left(\mathrm{~m}, 3 \mathrm{H}, \mathrm{H}_{\mathrm{d}, \mathrm{g}}\right) ;{ }^{13} \mathrm{C} \mathrm{NMR}\left(\mathrm{CDCl}_{3}, 100\right.$ $\mathrm{MHz}, \delta$ ): $65.6,112.2,118.7,120.4,120.5,122.2,123.5,123.7$, $127.5,127.7,128.1,128.5,131.4,132.1,138.6 .141 .6,144.8,146.0$, 149.4, 151.2. HRMS $\left(\mathrm{FAB}^{+}\right)$: calcd for $\mathrm{C}_{26} \mathrm{H}_{14}{ }^{79} \mathrm{BrN} 419.0310$. Found, 419.0305, $\mathrm{C}_{26} \mathrm{H}_{14}{ }^{81} \mathrm{BrN}$ 421.0289. Found 421.0300; Anal. Calcd for $\mathrm{C}_{25} \mathrm{H}_{14} \mathrm{BrN}$ : C, 74.30; H, 3.36; N, 3.33. Found: C, 74.44; $\mathrm{H}, 3.38$; N, 3.33 .

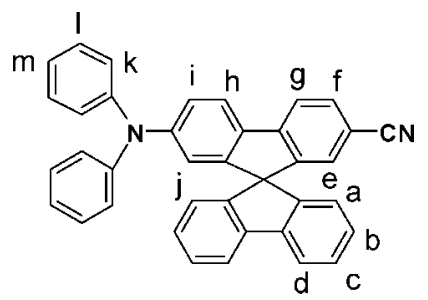

2.3.5. 2-Diphenylamino-7-cyano-9,9'-spirobifluorene (5). Dry toluene $(3 \mathrm{~mL})$ and tri-tert-butylphosphine $(3.8 \mathrm{~mL}, 0.19 \mathrm{mmol}, 0.05$ $\mathrm{M}$ in dry toluene) were added to a mixture of $4(0.2 \mathrm{~g}, 0.48 \mathrm{mmol})$, $\mathrm{Pd}(\mathrm{OAc})_{2},(11 \mathrm{mg}, 0.05 \mathrm{mmol})$, diphenylamine ( $\left.80 \mathrm{mg}, 0.48 \mathrm{mmol}\right)$, and $\mathrm{NaO}^{t} \mathrm{Bu}(0.05 \mathrm{~g}, 0.57 \mathrm{mmol})$, and the mixture was then heated under reflux for $18 \mathrm{~h}$ under argon. After cooling to room temperature, the solution was partitioned between $\mathrm{CH}_{2} \mathrm{Cl}_{2}$ and $\mathrm{H}_{2} \mathrm{O}$. The organic layer was dried $\left(\mathrm{MgSO}_{4}\right)$, filtered, and concentrated in vacuo. The residue was purified through column chromatography $\left(\mathrm{SiO}_{2} /\right.$ hexane-ethyl acetate $\left.85: 15\right)$ to afford $\mathbf{5}$ as a yellow solid (0.16 g, 67\%). mp $246-248{ }^{\circ} \mathrm{C}$. IR (neat) $v\left(\mathrm{~cm}^{-1}\right): 3066,2966$, $2226(-\mathrm{CN}), 1608(\mathrm{C}=\mathrm{C}), 1492 .{ }^{1} \mathrm{H} \mathrm{NMR}\left(\mathrm{CDCl}_{3}, 400 \mathrm{MHz}, \delta\right)$ : $6.53\left(\mathrm{~d}, J=2 \mathrm{~Hz}, 1 \mathrm{H}, \mathrm{H}_{\mathrm{j}}\right), 6.75\left(\mathrm{~d}, J=7.5 \mathrm{~Hz}, 2 \mathrm{H}, \mathrm{H}_{\mathrm{a}}\right), 6.88(\mathrm{~s}$, $\left.1 \mathrm{H}, \mathrm{H}_{\mathrm{e}}\right), 6.90-6.95\left(\mathrm{~m}, 6 \mathrm{H}, \mathrm{H}_{\mathrm{k}, \mathrm{m}}\right), 6.99(\mathrm{dd}, J=7.5,2 \mathrm{~Hz}, 1 \mathrm{H}$,
$\left.\mathrm{H}_{\mathrm{i}}\right), 7.08-7.16\left(\mathrm{~m}, 6 \mathrm{H}, \mathrm{H}_{\mathrm{b}, 1}\right), 7.35\left(\mathrm{t}, J=7.5 \mathrm{~Hz}, 2 \mathrm{H}, \mathrm{H}_{\mathrm{c}}\right), 7.58(\mathrm{~d}$, $\left.J=7.5 \mathrm{~Hz}, 1 \mathrm{H}, \mathrm{H}_{\mathrm{f}}\right), 7.67\left(\mathrm{~d}, J=8 \mathrm{~Hz}, 1 \mathrm{H}, \mathrm{H}_{\mathrm{h}}\right), 7.73-7.77(\mathrm{~m}$, $\left.3 \mathrm{H}, \mathrm{H}_{\mathrm{d}, \mathrm{g}}\right) .{ }^{13} \mathrm{C} \mathrm{NMR}\left(\mathrm{CDCl}_{3}, 100 \mathrm{MHz}, \delta\right): 65.7,109.2,118.2$, 119.0, 119.3, 120.0, 120.2, 121.4, 122.9, 123.3, 124.0, 127.1, 127.5, 127.9, 128.8, 131.7, 133.5, 141.2, 145.5, 146.6, 148.8, 149.1, 150.3. MS (EI) $\mathrm{m} / z$ (relative intensity): 421 (46), 340 (100), 341 (100), 162 (20). HRMS (EI): calcd for $\mathrm{C}_{38} \mathrm{H}_{24} \mathrm{~N}_{2}, 508.1939$; found, 508.1934. Anal. Calcd for $\mathrm{C}_{38} \mathrm{H}_{24} \mathrm{~N}_{2}$ : C, 89.74; H, 4.76; N, 5.51. Found: C, 89.68; H, 4.98; N, 5.51.

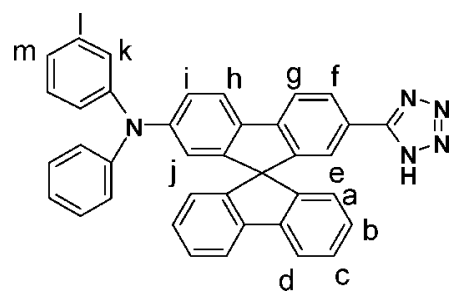

2.3.6. 2-Diphenylamino-7-tetrazolyl-9,9'-spirobifluorene (6). A mixture of the nitrile $5(0.43 \mathrm{~g}, 0.85 \mathrm{mmol})$, sodium azide $(0.17$ $\mathrm{g}, 2.54 \mathrm{mmol})$, and ammonium chloride $(0.14 \mathrm{~g}, 2.54 \mathrm{mmol})$ in dry DMF (6 mL) was heated at $100{ }^{\circ} \mathrm{C}$ (oil bath) for $19 \mathrm{~h}$ under argon. After cooling to room temperature, the solution was added dropwise to $3 \mathrm{~N}$ hydrochloric acid $(80 \mathrm{~mL})$, stirred vigorously for $10 \mathrm{~min}$, and then filtered. The filter cake was washed with water and dried in vacuo to afford 6 as a yellow solid $(0.43 \mathrm{~g}$, 92\%). mp 260-261 ${ }^{\circ} \mathrm{C}$. IR (KBr) $v\left(\mathrm{~cm}^{-1}\right): 3446(\mathrm{~N}-\mathrm{H}), 3057$, $1618(\mathrm{C}=\mathrm{C}), 1603,1582 .{ }^{1} \mathrm{H}$ NMR (DMSO- $\left.d_{6}, 400 \mathrm{MHz}, \delta\right)$ : $6.19\left(\mathrm{~s}, 1 \mathrm{H}, \mathrm{H}_{\mathrm{j}}\right), 6.73,\left(\mathrm{~d}, J=7.5 \mathrm{~Hz}, 2 \mathrm{H}, \mathrm{H}_{\mathrm{a}}\right), 6.85(\mathrm{~d}, J=7.5$ $\left.\mathrm{Hz}, 4 \mathrm{H}, \mathrm{H}_{\mathrm{k}}\right), 6.92-6.98\left(\mathrm{~m}, 3 \mathrm{H}, \mathrm{H}_{\mathrm{i}, \mathrm{m}}\right), 7.12-7.18\left(\mathrm{~m}, 6 \mathrm{H}, \mathrm{H}_{\mathrm{b}, \mathrm{l}}\right)$, $7.20\left(\mathrm{~s}, 1 \mathrm{H}, \mathrm{H}_{\mathrm{e}}\right), 7.38\left(\mathrm{t}, J=7.5 \mathrm{~Hz}, 2 \mathrm{H}, \mathrm{H}_{\mathrm{c}}\right), 7.95(\mathrm{~d}, J=7.5$ $\left.\mathrm{Hz}, 2 \mathrm{H}, \mathrm{H}_{\mathrm{d}}\right), 7.99$ (s, $\left.1 \mathrm{H}, \mathrm{H}_{\mathrm{h}}\right), 8.06-8.13\left(\mathrm{~m}, 2 \mathrm{H}, \mathrm{H}_{\mathrm{f}, \mathrm{g}}\right) .{ }^{13} \mathrm{C} \mathrm{NMR}$ (DMSO- $d_{6}, 100 \mathrm{MHz}, \delta$ ): 64.6, 116.2, 119.6, 119.7, 120.4, 121.3, $121.6,122.4,123.0,126.0,127.1,128.3,133.2,139.9,142.7$, 145.3, 146.1, 146.8, 147.8, 149.0. MS (EI) $\mathrm{m} / \mathrm{z}$ (relative intensity): $551\left(\mathrm{M}^{+}, 5\right), 523$ (20), 508 (100), 340 (20). HRMS (EI): calcd for $\mathrm{C}_{38} \mathrm{H}_{25} \mathrm{~N}_{5}$ 551.2110; found, 551.2122. Anal. Calcd for $\mathrm{C}_{38} \mathrm{H}_{25} \mathrm{~N}_{5}$ : C, 82.74; H, 4.57; N, 12.70. Found: C, 82.78; H, 4.64; N, 12.39 .

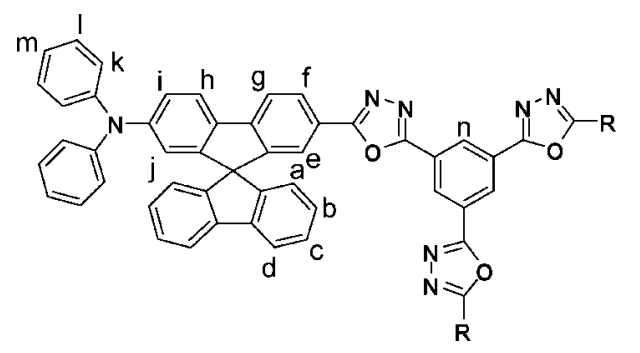

2.3.7. 1,3,5-Tris[7-diphenyl-(9,9'-spirobifluorene)-1,3,4-oxadiazoyl]benzene (OXD-DPA). 1,3,5-Benzenetricarboxylic acid chloride $(650 \mathrm{mg}, 0.19 \mathrm{mmol})$ was added in one portion to a solution of the tetrazole $6(0.36 \mathrm{~g}, 0.65 \mathrm{mmol})$ in dry toluene $(6 \mathrm{~mL})$, and the resulting mixture was then heated under reflux for $24 \mathrm{~h}$ under argon. After cooling to room temperature, the solution was concentrated in vacuo, and the residue was purified through column chromatography $\left(\mathrm{SiO}_{2} / \mathrm{CH}_{2} \mathrm{Cl}_{2}\right.$-ethyl acetate $\left.4: 1\right)$ to afford OXD-DPA as a yellow solid (0.22 g, 67\%). mp 268-270 ${ }^{\circ} \mathrm{C}$. IR (neat) $v\left(\mathrm{~cm}^{-1}\right): 3078,3049,1598(\mathrm{C}=\mathrm{C}), 1533,1468$. ${ }^{1} \mathrm{H} \mathrm{NMR}\left(\mathrm{CDCl}_{3}, 400 \mathrm{MHz}, \delta\right): 6.54\left(\mathrm{~d}, J=2 \mathrm{~Hz}, 3 \mathrm{H}, \mathrm{H}_{\mathrm{j}}\right)$, $6.81\left(\mathrm{~d}, J=7.5 \mathrm{~Hz}, 6 \mathrm{H}, \mathrm{H}_{\mathrm{a}}\right), 6.90-6.96\left(\mathrm{~m}, 18 \mathrm{H}, \mathrm{H}_{\mathrm{k}, \mathrm{m}}\right), 7.01$ $\left(\mathrm{dd}, J=7.5,2 \mathrm{~Hz}, 3 \mathrm{H}, \mathrm{H}_{\mathrm{i}}\right), 7.08-7.15\left(\mathrm{~m}, 18 \mathrm{H}, \mathrm{H}_{\mathrm{b}, 1}\right), 7.35(\mathrm{t}$, $\left.J=7.5 \mathrm{~Hz}, 6 \mathrm{H}, \mathrm{H}_{\mathrm{c}}\right), 7.47\left(\mathrm{~s}, 3 \mathrm{H}, \mathrm{H}_{\mathrm{e}}\right), 7.73(\mathrm{~d}, J=7.5 \mathrm{~Hz}, 3 \mathrm{H}$, $\left.\mathrm{H}_{\mathrm{h}}\right), 7.78\left(\mathrm{~d}, J=7.5 \mathrm{~Hz}, 6 \mathrm{H}, \mathrm{H}_{\mathrm{d}}\right), 7.89\left(\mathrm{~d}, J=7.5 \mathrm{~Hz}, 3 \mathrm{H}, \mathrm{H}_{\mathrm{g}}\right)$, $8.15\left(\mathrm{~d}, J=7.5 \mathrm{~Hz}, 3 \mathrm{H}, \mathrm{H}_{\mathrm{f}}\right), 8.80\left(\mathrm{~s}, 3 \mathrm{H}, \mathrm{H}_{\mathrm{n}}\right) .{ }^{13} \mathrm{C} \mathrm{NMR}\left(\mathrm{CDCl}_{3}\right.$, $100 \mathrm{MHz}, \delta): 65.8,118.8,119.7,120.4,121.2,121.3,122.6$, $123.0,123.3,123.6,124.1,125.9,127.1,127.3,127.7,127.9$, $129.0,134.4,141.7,145.6,147.1,147.4,148.7,149.6,151.1$, 
162.3, 165.5. HRMS (ESI ${ }^{+}$): calcd for $\mathrm{C}_{123} \mathrm{H}_{75} \mathrm{~N}_{9} \mathrm{O}_{3}$ (OXD-DP $+\mathrm{H})^{+}, 1726.6104$; found, 1726.6111. Anal. Calcd for $\mathrm{C}_{123} \mathrm{H}_{75} \mathrm{~N}_{9} \mathrm{O}_{3}$ : C, 85.49; H, 4.38; N, 7.30; O, 2.78. Found: C, 84.99; H, 4.39; N, 7.30.

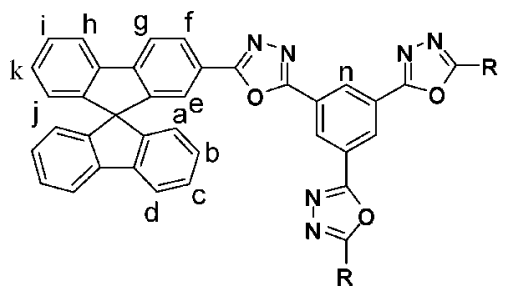

2.3.8. 1,3,5-Tris[2-(9,9'-spirobifluorene)-1,3,4-oxadiazoyl]benzene (OXD). 1,3,5-Benzenetricarboxylic acid chloride $(0.10 \mathrm{~g}, 0.38$ mmol) was added in one portion to a solution of 2-tetrazolyl-9, $9^{\prime}$ spirobifluorene $(0.45 \mathrm{~g}, 1.17 \mathrm{mmol})$ in dry toluene $(10 \mathrm{~mL})$, and the resulting mixture was then heated under reflux for $22 \mathrm{~h}$ under argon. After cooling to room temperature, the solution was concentrated in vacuo, and the residue was purified through column chromatography $\left(\mathrm{SiO}_{2} / \mathrm{CH}_{2} \mathrm{Cl}_{2}\right.$-ethyl acetate 9:1) to afford the title compound as a yellow solid $(0.23 \mathrm{~g}, 50 \%)$. mp $269-270{ }^{\circ} \mathrm{C}$. IR (neat) $v\left(\mathrm{~cm}^{-1}\right): 3072,3043,2919,1615(\mathrm{C}=\mathrm{C}), 1539 .{ }^{1} \mathrm{H}$ NMR $\left(\mathrm{CDCl}_{3}, 400 \mathrm{MHz}, \delta\right): 6.74\left(\mathrm{~d}, J=7.5 \mathrm{~Hz}, 6 \mathrm{H}, \mathrm{H}_{\mathrm{a}}\right), 6.77(\mathrm{~d}, J=$ $\left.7.5 \mathrm{~Hz}, 3 \mathrm{H}, \mathrm{H}_{\mathrm{j}}\right), 7.11\left(\mathrm{t}, J=7.5 \mathrm{~Hz}, 6 \mathrm{H}, \mathrm{H}_{\mathrm{b}}\right), 7.18(\mathrm{t}, J=7.5 \mathrm{~Hz}$, $\left.3 \mathrm{H}, \mathrm{H}_{\mathrm{k}}\right), 7.39\left(\mathrm{t}, J=7.5 \mathrm{~Hz}, 6 \mathrm{H}, \mathrm{H}_{\mathrm{c}}\right), 7.42\left(\mathrm{t}, J=7.5 \mathrm{~Hz}, 3 \mathrm{H}, \mathrm{H}_{\mathrm{i}}\right)$, $7.56\left(\mathrm{~d}, J=2 \mathrm{~Hz}, 3 \mathrm{H}, \mathrm{H}_{\mathrm{e}}\right), 7.88\left(\mathrm{~d}, J=7.5 \mathrm{~Hz}, 6 \mathrm{H}, \mathrm{H}_{\mathrm{d}}\right), 7.93(\mathrm{~d}$, $\left.J=7.5 \mathrm{~Hz}, 3 \mathrm{H}, \mathrm{H}_{\mathrm{g}}\right), 8.02\left(\mathrm{~d}, J=7.5 \mathrm{~Hz}, 3 \mathrm{H}, \mathrm{H}_{\mathrm{h}}\right), 8.21(\mathrm{dd}, J=$ 7.5, $\left.2 \mathrm{~Hz}, 3 \mathrm{H}, \mathrm{H}_{\mathrm{f}}\right), 8.83\left(\mathrm{~s}, 3 \mathrm{H}, \mathrm{H}_{\mathrm{n}}\right) .{ }^{13} \mathrm{C} \mathrm{NMR}\left(\mathrm{CDCl}_{3}, 100 \mathrm{MHz}\right.$, $\delta): 66.0,120.1,120.3,120.5,122.0,122.6,123.5,123.9,125.6$, 126.9, 127.0, 127.6, 127.7, 127.8, 128.9, 139.7, 141.5, 145.4, 147.0, 149.3, 149.4, 162.0, 165.0. HRMS (ESI ${ }^{+}$): calcd for $\mathrm{C}_{87} \mathrm{H}_{49} \mathrm{~N}_{6} \mathrm{O}_{3}$, 1225.3866; found, 1225.3843. Anal. Calcd for $\mathrm{C}_{87} \mathrm{H}_{48} \mathrm{~N}_{6} \mathrm{O}_{3} \cdot \mathrm{H}_{2} \mathrm{O}$ : C, 84.03; H, 4.06; N, 6.76. Found: C, 84.21; H, 4.11; N, 6.78.

2.4. Electrochemistry and Spectroelectrochemistry. All electrochemical and spectroelectrochemical studies were performed using dichloromethane $\left(\mathrm{CH}_{2} \mathrm{Cl}_{2}\right)$ solutions containing tetra- $n$ butylammonium perchlorate (TBAP) as the supporting electrolyte. Both materials were of reagent grade. $\mathrm{CH}_{2} \mathrm{Cl}_{2}$ was dried over $3 \AA$ molecular sieves for $48 \mathrm{~h}$ and used without further purification. TBAP was dried for $24 \mathrm{~h}$ under vacuum prior to use.

A conventional three-electrode electrochemical cell was used in the voltammetric experiments. The Pt wire working electrode (area: $2.16 \times 10^{-3} \mathrm{~cm}^{2}$ ) was polished with a $0.3 \mu \mathrm{m}$ alumina slurry, rinsed with a copious amount of triply distilled water, and then sonicated for $10 \mathrm{~min}$ to remove any residual polishing material. The counter electrode was a $\mathrm{Pt}$ coil. A silver (Ag) wire was used as a pseudoreference electrode. When the cyclic voltammogram $(\mathrm{CV})$ experiments were complete, ferrocene was added to the cell as an internal standard; all potentials are expressed relative to the ferrocene/ferrocenium $\left(\mathrm{Fc} / \mathrm{Fc}^{+}\right)$redox couple. The electrolyte was purged with dry argon before the electrochemical measurements, and the experiments were performed using an AUTOLAB PGSTAT30 potentiostat/galvanostat (general-purpose electrochemical system, GPES).

The spectroelectrochemical experiments were performed using a homemade cell built from a commercial UV-vis cuvette. An optically transparent indium tin oxide (ITO) electrode was used as the working electrode, a platinum wire was used as the counter electrode, and Ag wire was used as the pseudoreference electrode. The ITO electrode was carefully cleaned and dried prior to use. The cell was placed in the optical path of a Hewlett-Packard 8453 diode array spectrophotometer. Film thickness was measured using a Veeco Dektak $6 \mathrm{M}$ profilometer.

\section{Results and Discussion}

3.1. Synthesis. Scheme 2 depicts the synthesis of the CBZcapped star-shaped molecule OXD-CBZ. The synthesis began with the reaction of 2,7-dibromo-9, $9^{\prime}$-spirobifluorene with CBZ using Buchwald's N-arylation condition ${ }^{47}$ to give 2-bromo-7carbazoylspirobifluorene $(\mathbf{1})^{20}$ in $54 \%$ yield. The bromo group of $\mathbf{1}$ was reacted with $\mathrm{CuCN}$ to produce the 2-(carbazol-9-yl)7-cyano-9,9'-spirobifluorene (2) in an isolated yield of $82 \%$. Next, the cyano group in $\mathbf{2}$ was converted in high yield (92\%) to a tetrazole ring through a reaction with sodium azide. Treatment of the 2-(carbazol-9-yl)-7-tetrazol-1-yl-9,9'-spirobifluorene (3) with 1,3,5-benzenetricarbonyl trichloride afforded the target molecule OXD-CBZ in excellent yield (86\%).

The analogue OXD-DPA, which contains the same core structure but is end-capped with diphenylamino moieties, was synthesized following a similar synthetic pathway (Scheme 3). To facilitate the isolation of the unsymmetrical spirobifluorene derivative, we changed the reaction sequence slightly. In this case, 2,7-dibromo-9, $9^{\prime}$-spirobifluorene was reacted with $\mathrm{CuCN}$ to provide a readily separable product 2-bromo-7-cyano-9,9'spirobifluorene (4), which we converted to 2-diphenylamino7-cyano-9,9'-spirobifluorene (5) through Pd-catalyzed $\mathrm{C}-\mathrm{N}$ bond formation. OXD-DPA was then isolated in reasonably good yield after the same reactions as those presented for the synthesis of OXD-CBZ were performed. For comparison, we also synthesized the model compound 1,3,5-tris[2-(9, $9^{\prime}$-spirobifluorene)-1,3,4-oxadiazoyl]benzene (OXD), which lacks an electron-donating moiety.

3.2. Photophysical Properties. We recorded the absorption and emission spectra of these starburst molecules in several aprotic solvents; Table 1 summarizes the data. Figure 1 depicts the steady-state absorption and emission spectra of OXD-CBZ, OXD-DPA, and the core model compound OXD in various solvents. In $\mathrm{CH}_{2} \mathrm{Cl}_{2}, \mathbf{O X D}-\mathbf{C B Z}$ and OXD-DPA exhibited their absorption maxima centered at 356 and $401 \mathrm{~nm}$, respectively, which are significantly red-shifted relative to that of OXD (338 $\mathrm{nm}$ ), revealing that the $\pi$-conjugation lengths of $\mathbf{O X D}-\mathbf{C B Z}$ and OXD-DPA were effectively extended upon the introduction of the donor moieties (carbazole for OXD-CBZ, diphenylamino for OXD-DPA). We attribute the longer absorption wavelength of OXD-DPA to the better electron-donating character of its DPA groups, ${ }^{48,49}$ which are involved in effective electron delocalization with the bridging fluorene units. Nevertheless, the absorption spectra of OXD-CBZ and OXD-DPA exhibited limited dependence on the solvent polarity, indicating only weak electronic coupling between the donor (D; CBZ and diphenylamino groups) and acceptor (A; 1,3,4-oxadiazole) moieties in the ground state. Conversely, the emission maxima of the starburst bipolar molecules OXD-CBZ and OXD-DPA were strongly dependent on the solvent polarity. For example, OXDCBZ revealed an emission band maximized at $406 \mathrm{~nm}$ in benzene (excited at $361 \mathrm{~nm}$ ). Upon increasing the solvent polarity from benzene to acetonitrile, the emission band red shifted to $472 \mathrm{~nm}$. We observed similar behavior for OXDDPA in the same solvents: an emission band having its peak maximum at $444 \mathrm{~nm}$ in benzene and a very weak emission centered at $538 \mathrm{~nm}$ in $\mathrm{CH}_{3} \mathrm{CN}$. These results are strongly indicative of polarized excited states that we attribute to intramolecular charge transfer from the electron-donating groups to 1,3,4-oxadiazole moieties, which is supported by the solventpolarity-independent emission spectra of the model compound OXD. ${ }^{20,21,50}$ Instead of localizing their nitrogen atoms' lone pairs of electrons within the CBZ rings, the DPA moieties prefer to transfer these electrons to the electron-poor oxadiazole ring, which leads to a more polarized excited state. In addition, OXDCBZ and OXD-DPA exhibited fluorescence quantum yields in $\mathrm{CH}_{2} \mathrm{Cl}_{2}$, which were measured using an integration sphere, of 95 and $83 \%$, respectively, indicating that the rigid spirobifluorene skeleton was very beneficial for the radiation processes. Because of the polarized nature in the excited states of OXDCBZ and OXD-DPA, which were generated by intramolecular 
Scheme 1. Molecular Structures of Bipolar Starburst Monomers OXD-CBZ and OXD-DPA
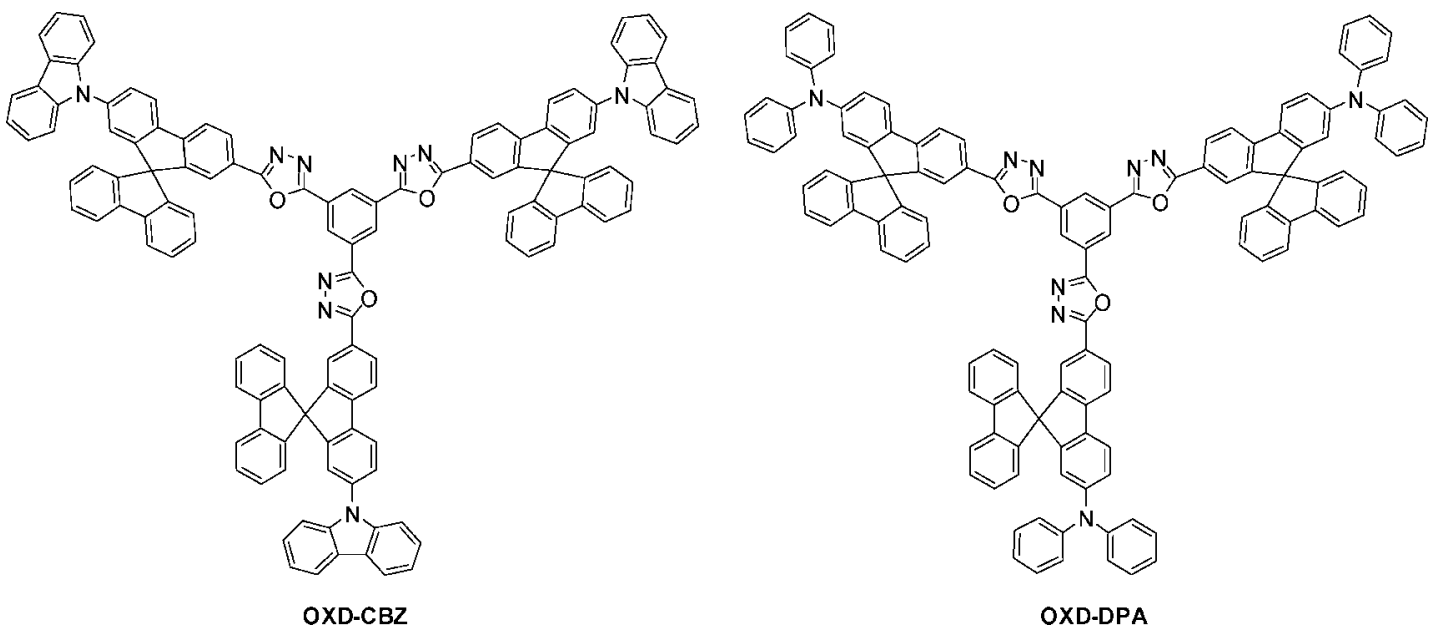

Scheme 2. Synthetic Pathway of Bipolar Starburst Compound OXD-CBZ
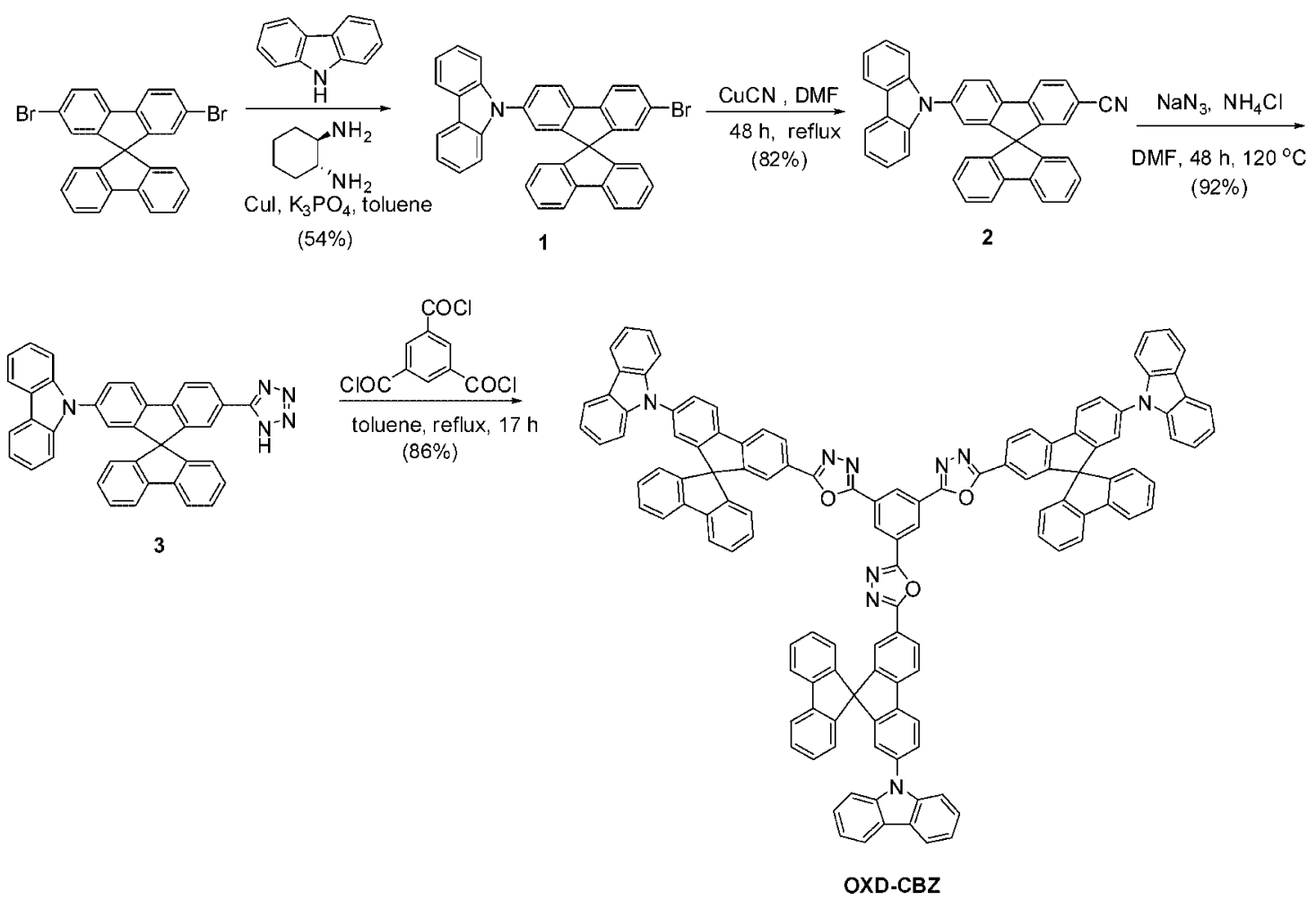

charge transfer, $\mathbf{O X D}-\mathbf{C B Z}$ and $\mathbf{O X D}-\mathbf{D P A}$ exhibited relatively low quantum yields of 42 and 3\% in acetonitrile, respectively.

3.3. Electrochemical Properties. Figure 2a presents a typical $\mathrm{CV}$ of OXD-CBZ in $0.1 \mathrm{M} \mathrm{TBAP} / \mathrm{CH}_{2} \mathrm{Cl}_{2}$. For the first positive potential scan, we observed an oxidation peak at ca. $0.881 \mathrm{~V}$ (peak I). From the first reverse negative potential scan, we detected two cathodic peaks: one at $0.796 \mathrm{~V}$ (peak II) and the other at $0.506 \mathrm{~V}$ (peak III). After the second scan, a new oxidation peak appeared at $0.616 \mathrm{~V}$ (peak IV) that was the complementary anodic process of peak III. In the meantime, peak I revealed an increased current and moved to a more anodic potential (peak V, $0.898 \mathrm{~V}$ ). The observation of a new oxidation couple (III-IV) in the second potential scan indicates that the OXD-CBZ radical cations were involved in very fast electrochemical/chemical reactions that produced a substance that was easier to oxidize than was the parent OXD-CBZ. In addition, when the potential was continuously cycled, we observed a progressive growth in all peak currents (Figure 2b). This behavior suggests that the oxidative coupling of the radical cations of OXD-CBZ produced a continuous build up of an electroactive and conductive layer on the electrode.

OXD-CBZ possesses two types of electroactive center that could be oxidized to generate an electroactive layer on the electrode surface: the terminal CBZ moieties and the spirobifluorene units. The electrochemical data reported in the literature reveal that the oxidation potential of fluorene derivatives is higher than that of the $\mathbf{C B Z}$ centers. ${ }^{16,20,38,51}$ In agreement with this fact, the model compound OXD (which lacks electrondonating moieties, Scheme 3) does not present oxidation peaks in this solvent-electrolyte electrochemical window. Therefore, we attribute the electrochemical processes observed at $0.881 \mathrm{~V}$ (peak I in Figure 2a) to the generation of CBZ radical cations, which subsequently dimerize to form 3,3'-bicarbazyl moieties. $^{20,37,48}$

The electrochemical properties of the analogue OXD-DPA are completely different. Figure 3 displays its reversible 
Scheme 3. Synthetic Pathway of Bipolar Starburst Monomer OXD-DPA and Model Compound OXD
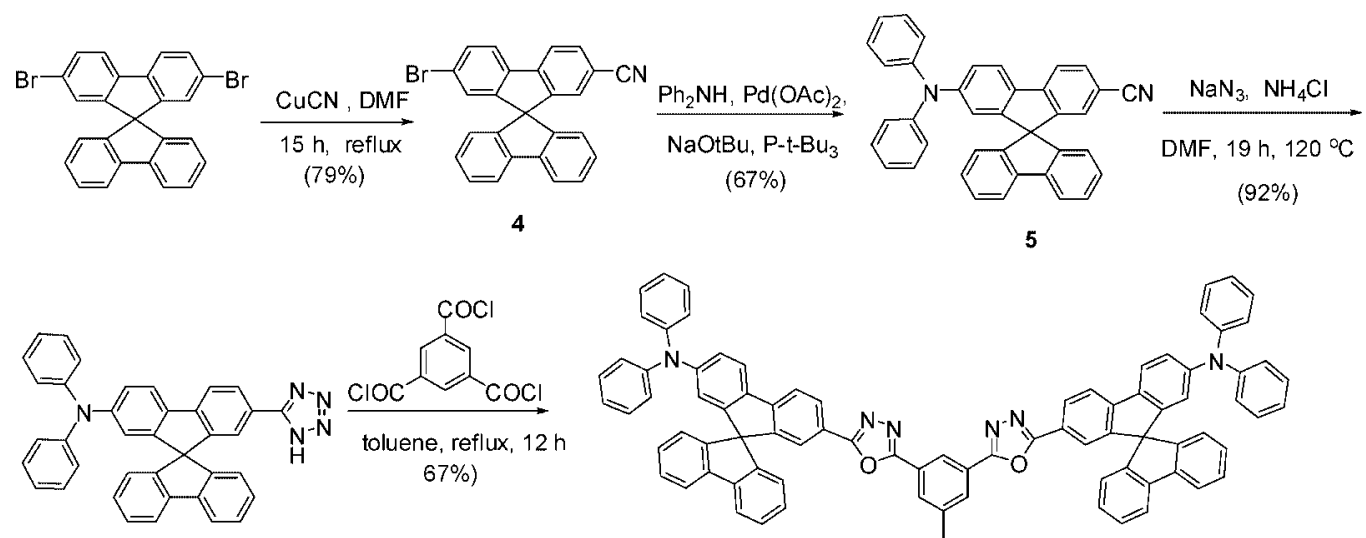

6

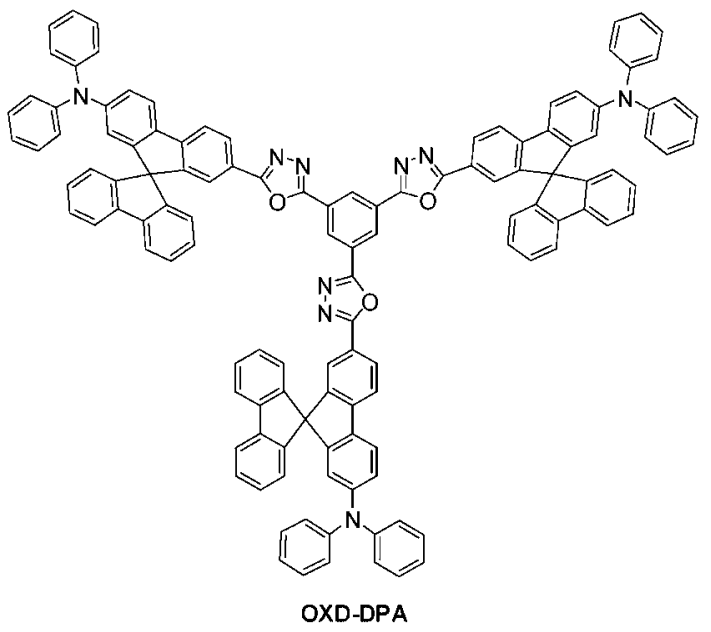

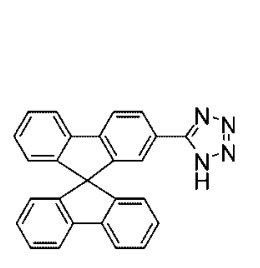

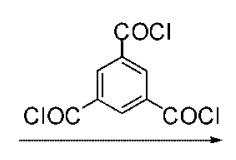

$(50 \%)$

Table 1. Absorption and Photoluminescence of Bipolar Compounds OXD-CBZ, OXD-DPA, and the Model Compound OXD in Various Solvents

\begin{tabular}{lrccc}
\hline compound & solvent & absorption $\left(\lambda_{\max }\right) \mathrm{nm}$ & $\mathrm{PL}\left(\lambda_{\max }\right) \mathrm{nm}^{a}$ & QY $(\%)^{b}$ \\
\hline OXD-CBZ & benzene & 361 & 406 & 91 \\
& $\mathrm{THF}^{c}$ & 357 & 442 & 99 \\
& $\mathrm{CH}_{2} \mathrm{Cl}_{2}$ & 356 & 448 & 95 \\
OXD-DPA & $\mathrm{CH}_{3} \mathrm{CN}$ & 350 & 472 & 42 \\
& benzene & 404 & 444 & 88 \\
& $\mathrm{THF}$ & 397 & 493 & 99 \\
& $\mathrm{CH}_{2} \mathrm{Cl}$ & 401 & 503 & 83 \\
& $\mathrm{CH}_{3} \mathrm{CN}$ & 392 & 538 & 3 \\
OXD & benzene $_{7}$ & 338 & 382 & 92 \\
& $\mathrm{THF}$ & 336 & 388 & 93 \\
& $\mathrm{CH}_{2} \mathrm{Cl}$ & 338 & 392 & 91 \\
& $\mathrm{CH}_{3} \mathrm{CN}$ & 334 & 402 & 87
\end{tabular}

${ }^{a}$ PL: photoluminescence. ${ }^{b} \mathrm{QY}$ : quantum yield measured with an integration sphere, which gave anthracene (in EtOH) with a quantum yield of $27 \%{ }^{c}$ THF: tetrahydrofuran.

electrochemical behavior $\left(E_{1 / 2}=0.486 \mathrm{~V}\right)$ with an $i_{\mathrm{pc}} / i_{\mathrm{pa}}$ (cathodic current peak/anodic current peak) ratio of close to one ${ }^{52}$ which indicates that the intermediates produced in the oxidation processes are stable on the time scale of the CV scan. Repetitive scans between 0 and $0.75 \mathrm{~V}$ provided the same patterns as those observed in the first scan. The curves were affected by only the diffusion contribution, and no new peaks were detected under these experimental conditions. This reversible oxidation process suggests that the radical cations of OXD-

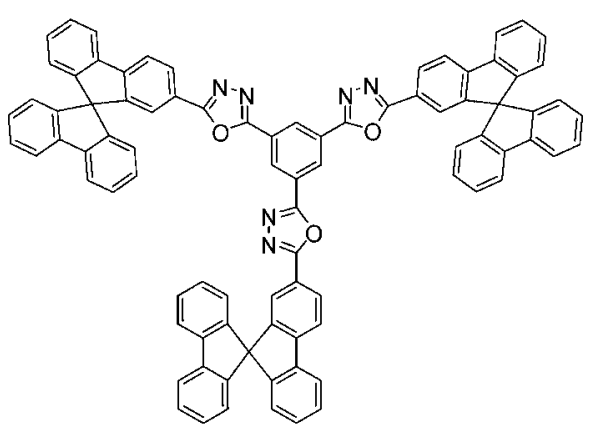

OXD

DPA are more stable than those produced from the oxidation of OXD-CBZ. As we observed in the photophysical studies, the DPA groups of OXD-DPA exhibited more-efficient $\pi$-delocalization, that is, a longer-wavelength absorption maximum, than did the CBZ groups of OXD-CBZ. Therefore, the radical cations of OXD-DPA are stabilized through resonance delocalization with the fluorene rings, making the subsequent dimerization process unfavorable. In contrast, the lone pair electrons of the nitrogen atom of a CBZ prefer to localize within the coplanar ring, making the oxidation potentials of $\mathrm{CBZ}$ derivatives normally independent of the nature of the $\mathrm{N} 9$ substituent. In general, CBZ moieties hold coupling rate constants that are 4 or 5 orders of magnitude higher than those of DPA units. ${ }^{20,48}$ It is fascinating that the electrochemical activity emanating from the same core of the starburst molecules OXD-CBZ and OXD-DPA was controlled by one carbon-carbon single bond connection at the peripheral groups.

3.3.1. OXD-CBZ Film Electrochemical Properties. To confirm the existence of an electroactive layer deposited on the electrode surface, we obtained modified electrodes by cycling the potential between 0 and $1.1 \mathrm{~V}$ under the same conditions as those previously used to obtain Figure 2 b. Herein, we refer to the electrode modified with the OXD-CBZ-derived polymeric thin film as ME-OXD-CBZ. We rinsed ME-OXD-CBZ with $\mathrm{CH}_{2} \mathrm{Cl}_{2}$ to remove any residual OXD-CBZ monomers and then transferred the new electrode to another cell containing an OXD- 
(a)

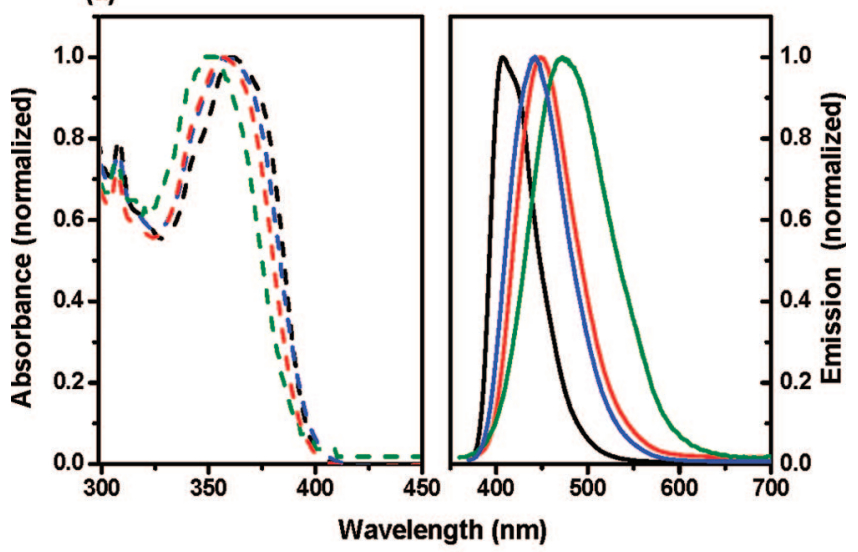

(b)

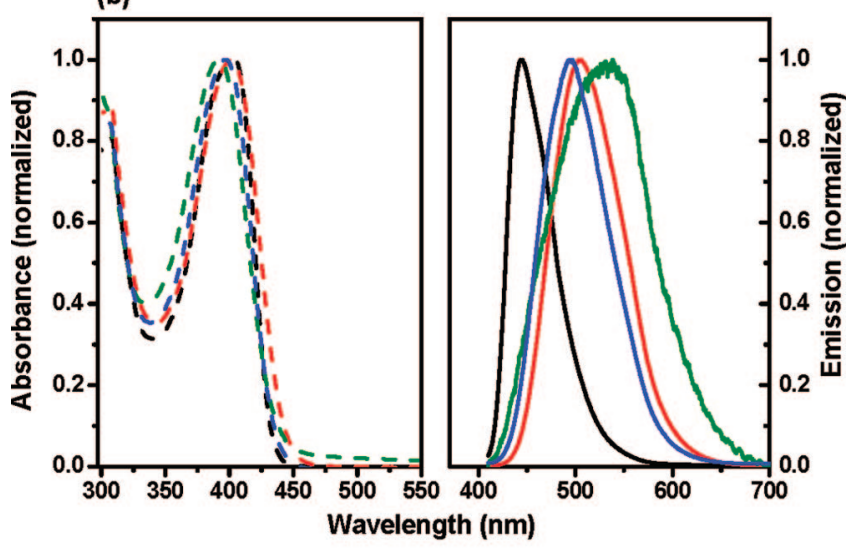

(c)

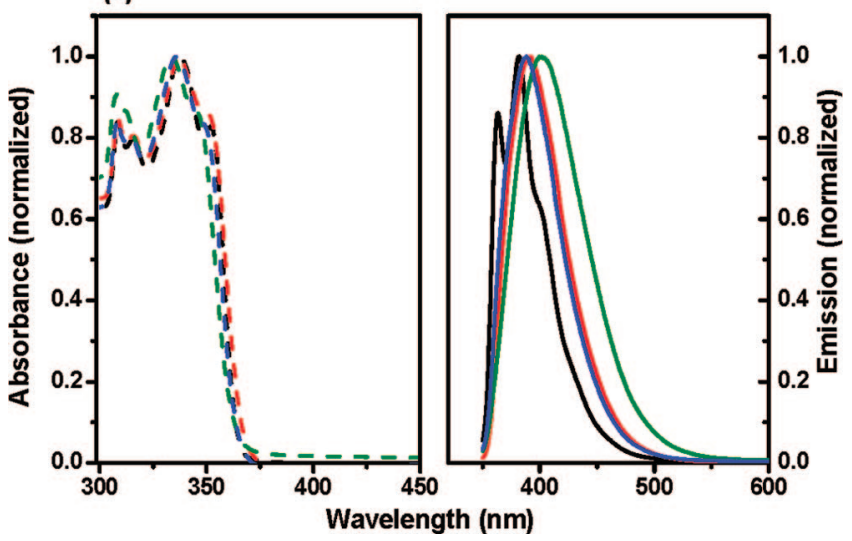

Figure 1. Absorption and photoluminescence spectra of (a) OXD-CBZ $\left(8.0 \times 10^{-6} \mathrm{M}\right)$, (b) OXD-DPA $\left(8.0 \times 10^{-6} \mathrm{M}\right)$, and (c) OXD $(8.0 \times$ $10^{-6} \mathrm{M}$ ) in various solvents (black: benzene; blue: tetrahydrofuran (THF); red: $\mathrm{CH}_{2} \mathrm{Cl}_{2}$; green: $\mathrm{CH}_{3} \mathrm{CN}$ ). The emission spectra were recorded upon excitation at the absorption maxima, respectively, in various solvents.

CBZ-free electrolyte solution. The redox processes of the MEOXD-CBZ are shown in the cyclic votammogram of Figure 4.

Figure 4 reveals two oxidation peaks at 0.653 and $0.877 \mathrm{~V}$ (peaks IV and V) and two associated cathodic waves at 0.572 and $0.803 \mathrm{~V}$ (peak systems IV/III and V/II, respectively). This observation confirms that the oxidation of CBZ units results in concomitant dimerization, leading to the formation of a stable electroactive film on the electrode surface. ${ }^{37,48,53}$ This behavior is similar to that observed for other N-substituted CBZ derivatives. ${ }^{48,54}$ In the present case, the rigid and branched structural features of OXD-CBZ force the dimerization to occur intermolecularly to form a cross-linked structure. ${ }^{20,21,37,48}$ Thus, we
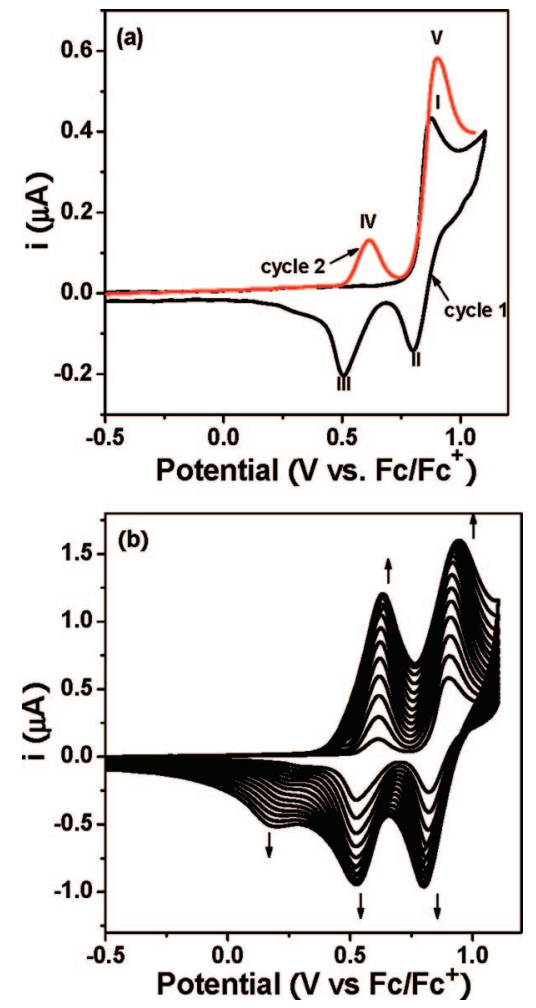

Figure 2. Cyclic voltammograms of $0.27 \mathrm{mM}$ OXD-CBZ in $0.1 \mathrm{M}$ $(n-\mathrm{Bu})_{4} \mathrm{NClO}_{4} / \mathrm{CH}_{2} \mathrm{Cl}_{2}$ at a scan rate of $0.050 \mathrm{~V} / \mathrm{s}$. (a) First and second cycles. (b) Repetitive cyclic voltammograms.

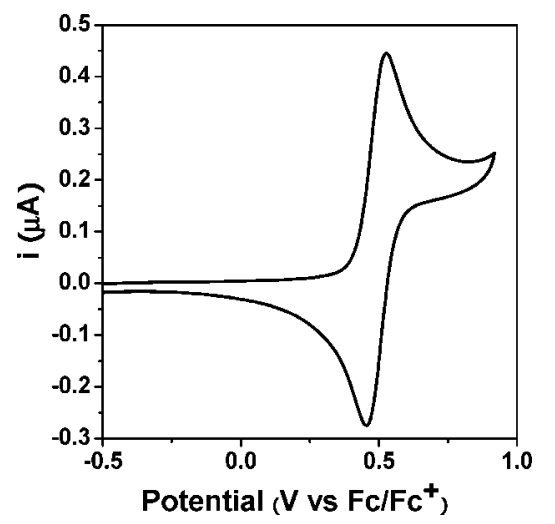

Figure 3. Cyclic voltammogram of $0.5 \mathrm{mM}$ OXD-DPA in $0.1 \mathrm{M}(n$ $\mathrm{Bu})_{4} \mathrm{NClO}_{4} / \mathrm{CH}_{2} \mathrm{Cl}_{2}$. Scan rate: $0.050 \mathrm{~V} / \mathrm{s}$.

assign the electrochemical processes in Figure 4 to the oxidation and reduction of the dimeric CBZ moieties. ${ }^{20,37,48,53}$ The first anodic peak corresponds to one-electron oxidations of the biscarbazole units to form radical cations, followed subsequently by the oxidation to dicationic species. ${ }^{5-56}$ When we compared the repetitive CV scans of the ME-OXD-CBZ thin film (Figure 4) with that of OXD-CBZ (Figure 2b), which was obtained in the presence of the monomer, we observed, however, that the wave at $0.279 \mathrm{~V}$ disappeared with progressing current. This behavior indicates that the low-potential wave corresponded to a reduction in the soluble products generated during the monomer oxidation process.

To analyze the growth and stability of the thin film, we investigated the relationship between the thin film oxidation charge $\left(Q_{\mathrm{a}, \mathbf{M E}-\mathbf{O X D}-\mathbf{C B Z}}\right)$ and the number of applied cycles. For each cycle, we measured the value of $Q_{\mathrm{a}, \mathbf{M E}-\mathbf{O X D}-\mathbf{C B Z}}$ of the thin film deposited on the electrode surface by integrating the anodic current of peaks IV and V. (See Figure 4.) Figure 5 presents a plot of $Q_{\mathrm{a}, \mathbf{M E}-\mathrm{OXD}-\mathrm{CBZ}}$ as a function of the number of scan cycles. 


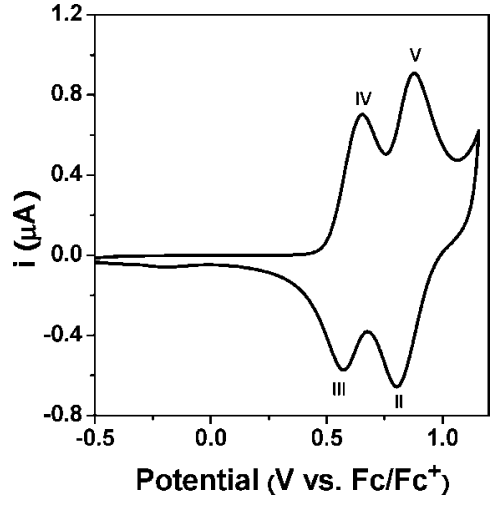

Figure 4. Cyclic voltammogram of ME-OXD-CBZ in $0.1 \mathrm{M}(n-$ $\mathrm{Bu})_{4} \mathrm{NClO}_{4} / \mathrm{CH}_{2} \mathrm{Cl}_{2}$. Scan rate: $0.050 \mathrm{~V} / \mathrm{s}$.

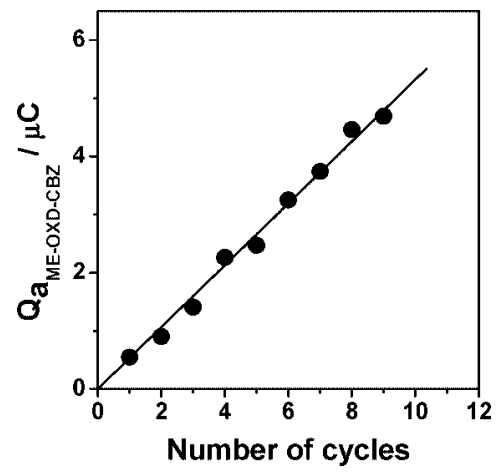

Figure 5. Anodic charge generated during the oxidation of ME-OXDCBZ versus the number of growth cycles. $0.27 \mathrm{mM} \mathrm{OXD-CBZ} \mathrm{in} 0.1$ $\mathrm{M}(n-\mathrm{Bu})_{4} \mathrm{NClO}_{4} / \mathrm{CH}_{2} \mathrm{Cl}_{2}$. Scan rate: $0.050 \mathrm{~V} / \mathrm{s}$.

A linear relationship (correlation coefficient: $r=0.996$ ) existed between the amount of deposited material and the number of scan cycles, indicating that the resulting electroactive film grew homogeneously until a film thicknesses of $580 \AA$ (Figure 5) was achieved. This is an evidence of good conductivity and strong adherence to the metallic electrode. Moreover, we estimated (from the oxidation charge, electrode area, and film thickness) an active redox site concentration of $\sim 2.2$ $\mathrm{mol} \cdot \mathrm{dm}^{-3}$, which remains approximately constant for different film thicknesses. The homogeneous formation of polymeric films through electrochemical techniques allows great control over the film thickness, which is an important parameter in the preparation of optoelectronic devices. Furthermore, we correlated the total oxidation charge $\left(Q_{\mathrm{a}, \mathrm{T}}\right)$ involved in each cycle of film growth with the film oxidation charge $\left(Q_{\mathrm{a}, \mathrm{ME}-\mathrm{OxD}-\mathrm{CBZ}}\right)$. For each cycle, we obtained the value of $Q_{\mathrm{a}, \mathrm{T}}$ through integration of the anodic sweep in the voltammogram presented in Figure 2a. The total oxidation charge was the sum of the diffusional charge $Q_{\mathrm{a}, \mathbf{O X D} \text {-CBZ}}$, corresponding to the oxidation of the OXDCBZ monomer, and the nondiffusional charge $Q_{\mathrm{a}, \mathrm{ME}-\mathrm{OXD}-\mathrm{CBZ}}$, which was related to the degree of oxidation of the film (eq 1)

$$
Q_{\mathrm{a}, \mathrm{T}}=Q_{\mathrm{a}, \mathrm{OXD}-\mathrm{CBZ}}+Q_{\mathrm{a}, \mathrm{ME}-\mathrm{OXD}-\mathrm{CBZ}}
$$

$Q_{\mathrm{a}, \text { ME-OXD-CBZ linearly correlates with the number of forma- }}$ tion cycles (Figure 5), which indicates that the diffusional charge $\left(Q_{\mathrm{a}, \mathrm{OXD}-\mathrm{CBZ}}\right)$ involved in each formation cycle is nearly constant. As we described above, the film formation mechanism involved the oxidative coupling of two CBZ radical cations (two electrons). The oxidation of the film (in the potential range used

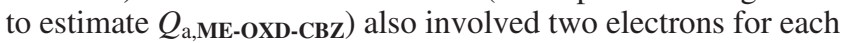
active site: the oxidation of biscarbazole units to form dications. $^{20,48,54}$ Therefore, we can estimate $Q_{\mathrm{a}, \text { OXD-CBZ }}$ from the intercept of the plot of $Q_{\mathrm{a}, \mathrm{T}}$ with respect to $Q_{\mathrm{a}, \mathbf{M E}-\mathbf{O X D}-\mathrm{CBZ}}$ (Figure

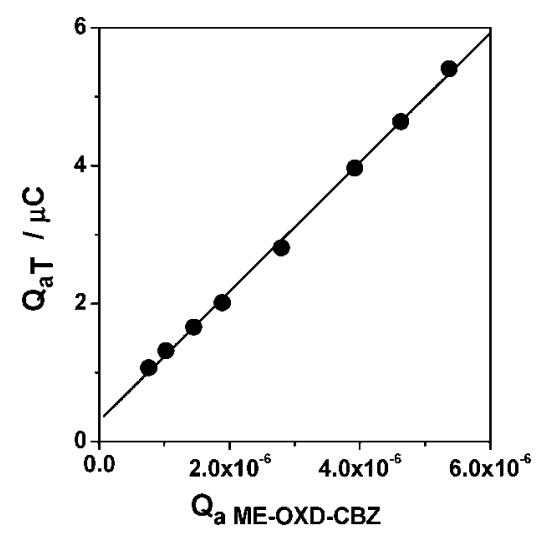

Figure 6. Plot of $Q_{\mathrm{a}, \mathrm{T}}$ versus $Q_{\mathrm{a}, \mathbf{M E}-\mathrm{OXD}-\mathrm{CBZ}}$ (eq 1 ).

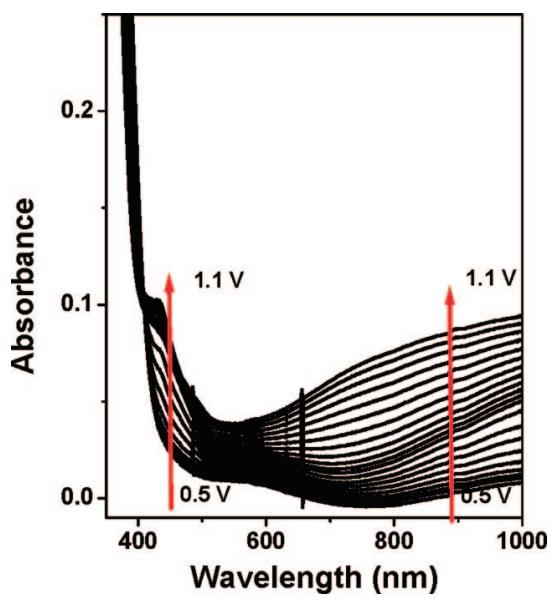

Figure 7. UV-vis spectra of thin films derived from $\mathbf{O X D}-\mathbf{C B Z}$ on an ITO electrode at various potentials. $(n-\mathrm{Bu})_{4} \mathrm{NClO}_{4} / \mathrm{CH}_{2} \mathrm{Cl}_{2}(0.1 \mathrm{M})$ was used as the supporting electrolyte. Scan rate: $0.025 \mathrm{~V} / \mathrm{s}$.

6). The observed linear behavior in Figure 6 indicates that the film is formed in high yield from the electrogenerated radical cations. However, the slope is slightly less than 1, which implies that not all of the charges used for monomer oxidation were involved in the film formation process. Therefore, we suspect that a soluble product was formed during the electropolymerization reaction; this result echoes our conclusion from the analysis of Figure $2 b$ and the progressing waves at $0.279 \mathrm{~V}$.

3.4. Spectroelectrochemical Analysis of ME-OXD-CBZ. We confirmed the presence of biscarbazole moieties as electroactive centers in the cross-linked film structure through spectroelectrochemical studies conducted on the resulting thin film. We grew this film over a semitransparent ITO electrode by cycling the potential between 0 and $1.0 \mathrm{~V}$ in the presence of OXD-CBZ $(0.1 \mathrm{M})$ in $0.1 \mathrm{M} \mathrm{TBAP} / \mathrm{CH}_{2} \mathrm{Cl}_{2}$ solution. Figure 7 shows a progression of electronic absorption spectra of this modified ITO electrode upon applied potential variation.

At applied potentials of $<0.5 \mathrm{~V}$, the film is in its neutral form where two absorption peaks at 305 and $348 \mathrm{~nm}$ (not shown) are detected, which we attribute to the $\pi-\pi^{*}$ transitions of the biscarbazole ${ }^{37,54,57,58}$ and the fluorene-containing core ${ }^{58,59}$ (cf. Figure 1), respectively. Consequently, the film is almost transparent in the visible region. When the applied potential became more anodic, the spectra displayed one absorption peak at ca. $440 \mathrm{~nm}$ and a broadband that extended to the near-IR range (Figure 7), which we assigned to the formation of the biscarbazole radical cation and dication, ${ }^{20,56}$ respectively. Although a broadband centered at ca. $800 \mathrm{~nm}$ is often observed in CBZ polymers, ${ }^{20,56}$ in the case of our electrodeposited OXDCBZ thin films, we observed a bathochromic shift in the near- 

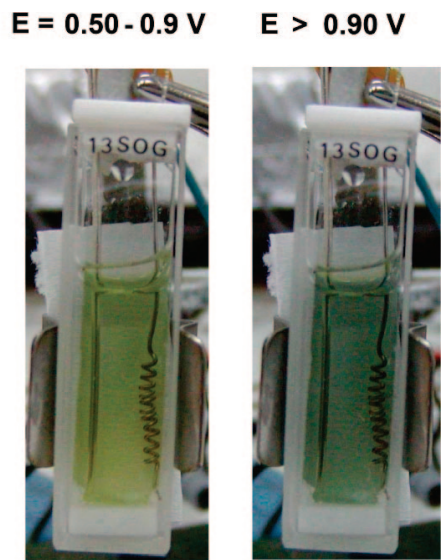

Figure 8. Photographic images of a film derived from OXD-CBZ on an ITO electrode at various applied potentials.
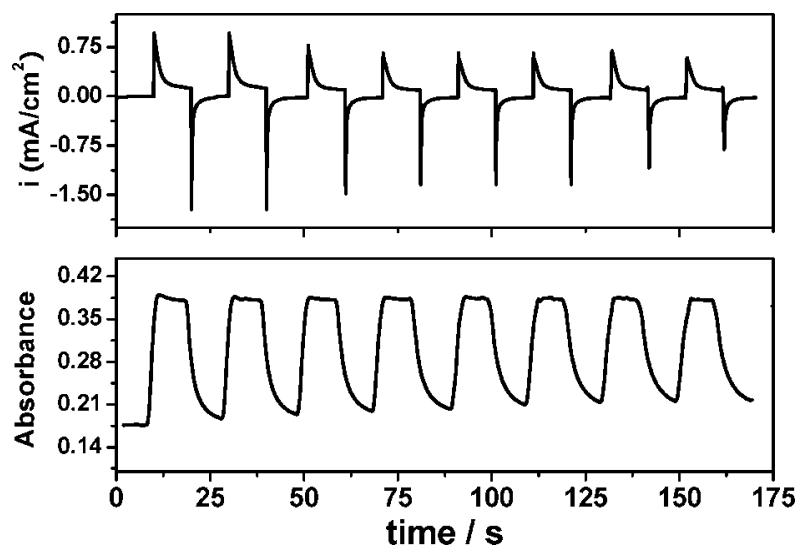

Figure 9. Electrochromic switching, current, and optical response to potential steps between 0.0 and $0.8 \mathrm{~V}$ applied to ME-OXD-CBZ in $0.1 \mathrm{M}(n-\mathrm{Bu})_{4} \mathrm{NClO}_{4} / \mathrm{CH}_{2} \mathrm{Cl}_{2}$.

IR range, which indicated that a more stable charged moiety was present in the film structure. These facts are in agreement with the presence of a conducting polymer with good charge transport capability. The observed UV-vis absorption changes of the OXD-CBZ-derived film were fully reversible upon varying the applied potential. In addition, they were associated with significant color changes (from transparent to green to light blue) that were homogeneous across the ITO electrode surface and easy to detect with the naked eye (Figure 8).

Electrochromic parameters of ME-OXD-CBZ were obtained from the current-time and absorbance-time profiles recorded upon repetitive potential steps between 0.0 and $0.8 \mathrm{~V}$ at $0.1 \mathrm{~Hz}$ frequency $^{60}$ (Figure 9).

The response time required for $90 \%$ full-transmittance $(T)$ change at $\lambda=435 \mathrm{~nm}$ was $2.5 \mathrm{~s}$ for the coloration step. The coloration efficiency $\eta=\Delta A(\lambda) / Q$ (where $Q$ is the injected charge per unit electrode area and $\Delta A$ is the change in absorbance at $\lambda=435 \mathrm{~nm}$ ) yielded coloration efficiency of $\sim 85$ $\mathrm{C}^{-1} \cdot \mathrm{cm}^{2}$. The results are comparable to those of other structurally related electrochromic polymers ${ }^{20,21,61,62}$ and show that the electropolymerized film holds fairly good electrochromic characteristics.

\section{Conclusions}

We have synthesized new bipolar starburst monomers featuring an electron-deficient core bridged by rigid spirobifluorenes to electroactive termini. These monomers exhibit distinct photoinduced charge transfer emission, as evidenced by the photoluminescence being dependent on the solvent polarity.
More interestingly, the bipolar character and the intramolecular redox gradient allowed us to prepare a new hole-conducting polymer through electropolymerization. The electropolymerization process was dependent upon the structural features of the end-capping groups. The CBZ-terminated OXD-CBZ underwent efficient electropolymerization in a high yield because the OXD-CBZ radical cations were localized within the CBZ ring and because the $\mathrm{C} 3$ and $\mathrm{C} 6$ positions of the $\mathrm{CBZ}$ were substituent-free, leading to effective cross-linking. In contrast, the DPA-terminated OXD-DPA produced very stable radical cations upon electro-oxidation; they did not undergo any associated electrochemical process. The higher stability of the radical cations of OXD-DPA through resonance stabilization with the fluorene rings impeded the dimerization reactions typical of triphenylamine. The electrogenerated OXD-CBZderived polymer films were electrochemically homogeneous and exhibited good conductivity properties and strong adherence to the metallic electrode. They also underwent reversible and significant color changes under different applied potentials. The film colors (transparent, green, and light blue) were homogeneous across the ITO electrode surface and were easily detected by the naked eye.

Acknowledgment. We thank Consejo Nacional de Investigaciones Científicas y Técnicas (CONICET-Argentina), Agencia Nacional de Promoción Científica y Tecnológica (ANPCYTArgentina), Secretaría de Ciencia y Técnica de la Universidad Nacional de Río Cuarto (SECYT-UNRC), and the National Science Council of Taiwan for financial support. L.O. and F.F. are scientific members of CONICET.

Supporting Information Available: Assignments of ${ }^{13} \mathrm{C}$ NMR spectra for new compounds, ${ }^{1} \mathrm{H}$ and ${ }^{13} \mathrm{C}$ NMR spectra of compounds CBZ-OXD, DPA-OXD, and OXD, and ${ }^{1} \mathrm{H}-{ }^{-1} \mathrm{H}$ COSY of CBZOXD. This material is available free of charge via the Internet at http://pubs.acs.org.

\section{References and Notes}

(1) Dendrimers and Other Dendritic Polymers; Frèchet, J. M. J., Tomalia, D. A., Eds.; Wiley: New York, 2001.

(2) Marcos, M.; Rapún, R. M.; Omenat, A.; Serrano, J. L. Chem. Soc. Rev. 2007, 36, 1889-1901.

(3) Lo, S.-C.; Burn, P. L. Chem. Rev. 2007, 107, 1097-1116.

(4) Kwon, T.-W.; Alam, M. M.; Jenekhe, S. A. Chem. Mater. 2004, 16, 4657-4666.

(5) Thelakkat, M. Macromol. Mater. Eng. 2002, 287, 442-461.

(6) Shirota, Y. J. Mater. Chem. 2000, 10, 1-25.

(7) Burn, P. L.; Lo, S.-C.; Samuel, I. D. Adv. Mater. 2007, 19, 16751688.

(8) Adhikari, R. M.; Mondal, R.; Shah, B. K.; Neckers, D. C. J. Org. Chem. 2007, 72, 4727-4732.

(9) Lu, J.; Xia, P. F.; Lo, P. K.; Tao, Y.; Wong, M. S. Chem. Mater. 2006, 18, 6194-6203.

(10) Nakashima, T.; Satoh, N.; Albrecht, K.; Yamamoto, K. Chem. Mater. 2008, 20, 2538-2543.

(11) Cuadrado, I.; Casado, M.; Moran, M.; Losada, J.; Belsky, V. J. Am. Chem. Soc. 1997, 119, 7613-7614.

(12) Valerio, C.; Fillaut, J. L.; Ruiz, J.; Guittard, Z. J.; Blais, J. C.; Astruc, D. J. Am. Chem. Soc. 1997, 119, 2588-2589.

(13) Zhao, L.; Li, C.; Zhang, Y.; Zhu, X.-H.; Peng, J.; Cao, Y. Macromol. Rapid Commun. 2006, 27, 914-920.

(14) Guldi, D. M.; Swartz, A.; Chuping, L.; Gómez, R.; Segura, J. L.; Martin, N. J. Am. Chem. Soc. 2002, 124, 10875-10886.

(15) Xia, H.; He, J.; Peng, P.; Zhou, Y.; Li, Y.; Tian, W. Tetrahedron Lett. 2007, 48, 5877-5881.

(16) Son, H.-J.; Han, W.-S.; Lee, K.-H.; Jung, H.-J.; Lee, C.; Ko, J.; Kang, S.-O. Chem. Mater. 2006, 18, 5811-5813.

(17) Taranekar, P.; Fulghum, T.; Patton, D.; Ponnapati, R.; Clyde, G.; Advincula, R. J. Am. Chem. Soc. 2007, 129, 12537-12548.

(18) Hughes, G.; Bryce, M. R. J. Mater. Chem. 2005, 15, 94-107.

(19) Baba, A.; Onishi, K.; Knoll, W.; Advincula, R. C. J. Phys. Chem. B 2004, 108, 18949-18955.

(20) Natera, J.; Otero, L.; Sereno, L.; Fungo, F.; Wang, N.-S.; Tsai, Y.M.; Hwu, T.-Y.; Wong, K.-T. Macromolecules 2007, 40, 4456-4463. 
(21) Otero, L.; Sereno, L.; Fungo, F.; Liao, Y.-L.; Lin, C.-Y.; Wong, K.T. Chem. Mater. 2006, 18, 3495-3502.

(22) Promarak, V.; Ichikawa, M.; Sudyoadsuk, T.; Saengsuwan, S.; Jungsuttiwong, S.; Keawin, T. Synth. Met. 2007, 157, 17-22.

(23) Nantalaksakul, A.; Reddy, D.; Bardeen, C.; Thayumanavan, S. Photosynth. Res. 2006, 87, 133-150.

(24) Burn, P. L.; Beavington, R.; Frampton, M. J.; Pillow, J. N. G.; Halim, M.; Lupton, J. M.; Samuel, I. D. W. Mater. Sci. Eng., B 2001, 85, 190-194.

(25) Martinez, F. J.; González, B.; Alonso, B.; Losada, J.; Garcia-Armada, M. P.; Casado, C. M. J. Inorg. Organomet. Polym. 2008, 18, 51-58.

(26) Taranekar, P.; Fulghum, T.; Baba, A.; Patton, D.; Advincula, R. Langmuir 2007, 23, 908-917.

(27) Percec, V.; Barboiu, B.; Grigoras, C.; Bera, T. K. J. Am. Chem. Soc. 2003, 125, 6503-6516.

(28) Taranekar, P.; Park, J.-Y.; Patton, D.; Fulghum, T.; Ramon, G. J.; Advincula, R. Adv. Mater. 2006, 18, 2461-2465.

(29) Albrecht, K.; Yamamoto, K. J. Photopolym. Sci. Technol. 2006, 19, $175-176$.

(30) Kimoto, A.; Cho, J.-S.; Ito, K.; Aoki, D.; Miyake, T.; Yamamoto, K. Macromol. Rapid Commun. 2005, 26, 597-601.

(31) Peng, Q.; Li, M.; Lu, S.; Tang, X. Macromol. Rapid Commun. 2007, 28, 785-791.

(32) Yang, C.-H.; Liu, F.-J.; Huang, L.-R.; Wang, T.-L.; Lin, W.-C.; Sato, M.; Chen, C.-H.; Chang, C.-C. J. Electroanal. Chem. 2008, 617, 101110 .

(33) Shirota, Y.; Kageyama, H. Chem. Rev. 2007, 107, 953-1010.

(34) Li, F.; Chen, Z.; Qu, B.; Wei, W.; Gong, Q. J. Phys. D: Appl. Phys. 2005, 38, 847-851.

(35) Wong, K.-T.; Lin, Y.-H.; Wu, H.-H.; Fungo, F. Org. Lett. 2007, 9 , 4531-4534.

(36) Lu, S.; Liu, T.; Ke, L.; Ma, D.-G.; Chua, S.-J.; Huang, W. Macromolecules 2005, 38, 8494-8502.

(37) Morin, J.-F.; Leclerc, M.; Ade, D.; Siove, A. Macromol. Rapid Commun. 2005, 26, 761-778.

(38) Fungo, F.; Wong, K.-T.; Ku, S.-Y.; Hung, Y.-Y.; Bard, A. J. J. Phys. Chem. B 2005, 109, 3984-3989.

(39) Oyamada, T.; Chang, C.-H.; Chao, T.-C.; Fang, F.-C.; Wu, C.-C.; Wong, K.-T.; Sasabe, H.; Adachi, C. J. Phys. Chem. C 2007, 111, 108-115.

(40) Wu, C.-C.; Liu, W.-G.; Hung, W.-Y.; Liu, T.-L.; Lin, Y.-T.; Lin, H.W.; Wong, K.-T.; Chien, Y.-Y.; Chen, R.-T.; Hung, T.-H.; Chao, T.C.; Chen, Y.-M. Appl. Phys. Lett. 2005, 87, 052103-1-052103-3.

(41) Zeng, G.; Chua, S.-J.; Huang, W. Thin Solid Films 2002, 417, 194197.
(42) Markham, J. P. J.; Namdas, E. B.; Anthopoulos, T. D.; Samuel, I. D. W.; Richards, G. J.; Burn, P. L. Appl. Phys. Lett. 2004, 85, 14631465.

(43) Lai, W.-Y.; Zhu, R.; Fan, Q.-L.; Hou, L.-T.; Cao, Y.; Huang, W. Macromolecules 2006, 39, 3707-3709.

(44) Levermore, P. A.; Xia, R.; Lai, W.; Wang, X. H.; Huang, W.; Bradley, D. D. C. J. Phys. D: Appl. Phys. 2007, 40, 1896-1901.

(45) Li, Z.; Di, C.; Zhu, Z.; Yu, G.; Li, Z.; Zeng, Q.; Li, Q.; Liu, Y.; Qin, J. Polymer 2006, 47, 7889-7899.

(46) Li, Z. H.; Wong, M. S. Org. Lett. 2006, 8, 1499-1502.

(47) Klapars, A.; Antilla, J. C.; Huang, X.; Buchwald, S. L. J. Am. Chem. Soc. 2001, 123, 7727-7729.

(48) Ambrose, J. F.; Carpenter, L. L.; Nelson, R. F. J. Electrochem. Soc. 1975, 122, 876-877.

(49) Seo, E. T.; Nelson, R. F.; Fritsch, J. M.; Marcoux, L. S.; Leedy, D. W.; Adams, R. N. J. Am. Chem. Soc. 1966, 88, 3498-3503.

(50) Ku, S.-Y.; Cheng, Y.-M.; Lin, X.-Y.; Hung, Y.-Y.; Pu, S.-C.; Wong, K.-T.; Chou, P.-T.; Lee, G.-H. J. Org. Chem. 2006, 71, 456-465.

(51) Inaoka, S.; Roitman, D. B.; Advincula, R. C. Chem. Mater. 2005, 17, 6781-6789.

(52) Bard, A. J.; Faulkner, L. R. Electrochemical Methods: Fundamentals and Applications; Wiley: New York, 1980.

(53) Ambrose, J. F.; Nelson, R. F. J. Electrochem. Soc. 1968, 115, 11591164.

(54) Tran-Van, F.; Henri, T.; Chevrot, C. Electrochim. Acta 2002, 47, 29272936.

(55) Siove, A.; Ades, D.; N'gbilo, E.; Chevrot, C. Synth. Met. 1990, 38, $331-340$.

(56) Marrec, P.; Dano, C.; Gueguen-Simonet, N.; Simonet, J. Synth. Met. 1997, 89, 171-179.

(57) Wei, Z. H.; Xu, J. K.; Nie, G. M.; Du, Y. K.; Pu, S. Z. J. Electroanal. Chem. 2006, 589, 112-119.

(58) Li, M.; Tang, S.; Shen, F.; Liu, M.; Xie, W.; Xia, H.; Liu, L.; Tian, L.; Xie, Z.; Lu, P.; Hanif, M.; Lu, D.; Cheng, G.; Ma, Y. J. Phys. Chem. B 2006, 110, 17784-17789.

(59) Chien, Y.-Y.; Wong, K.-T.; Chou, P.-T.; Cheng, Y.-M. Chem. Commun. 2002, 2874-2875.

(60) Monk, P. M. S.; Mortimer, R. J.; Rosseinsky, D. R. Electrochromism: Fundamentals and Applications, VCH: Weinheim, Germany, 1995.

(61) Liou, G.-S.; Chen, H.-W.; Yen, H.-J. J. Polym. Science, Part A: Polym. Chem. 2006, 44, 4108-4121.

(62) Liou, G.-S.; Chen, H.-W.; Yen, H.-J. Macromol. Chem. Phys. 2006, 207, 1589-1598.

MA801865Y 